\title{
MAXIMAL SUBGROUPS AND IRREDUCIBLE REPRESENTATIONS OF GENERALISED MULTI-EDGE SPINAL GROUPS
}

\author{
BENJAMIN KLOPSCH AND ANITHA THILLAISUNDARAM
}

\begin{abstract}
Let $p \geq 3$ be a prime. A generalised multi-edge spinal group

$$
G=\left\langle\{a\} \cup\left\{b_{i}^{(j)} \mid 1 \leq j \leq p, 1 \leq i \leq r_{j}\right\}\right\rangle \leq \operatorname{Aut}(T)
$$

is a subgroup of the automorphism group of a regular $p$-adic rooted tree $T$ that is generated by one rooted automorphism $a$ and $p$ families $b_{1}^{(j)}, \ldots, b_{r_{j}}^{(j)}$ of directed automorphisms, each family sharing a common directed path disjoint from the paths of the other families.

This notion generalises the concepts of multi-edge spinal groups, including the widely studied GGS-groups, and extended Gupta-Sidki groups that were introduced by Pervova. Extending techniques that were developed in these more special cases, we prove: generalised multiedge spinal groups that are torsion have no maximal subgroups of infinite index. Furthermore we use tree enveloping algebras, which were introduced by Sidki and Bartholdi, to show that certain generalised multi-edge spinal groups admit faithful infinite dimensional irreducible representations over the prime field $\mathbb{Z} / p \mathbb{Z}$.
\end{abstract}

\section{INTRODUCTION}

Throughout let $p$ be an odd prime, and let $T$ denote a regular $p$-adic rooted tree. Pioneering constructions of Grigorchuk, Gupta and Sidki in the 1980s led to the first examples of subgroups of the automorphism group $\operatorname{Aut}(T)$ that are now called GGS-groups. Since then the profinite group $\operatorname{Aut}(T)$ has become a 'building site' for finitely generated, residually finite groups with interesting properties, in particular branch groups. Typically the groups are realised as subgroups of $\operatorname{Aut}(T)$ that are generated by tree automorphisms with built-in self-similarities; see [4, 13].

In this paper we consider a collection $\mathscr{C}$ of subgroups of $\operatorname{Aut}(T)$, called generalised multi-edge spinal groups, that form a common generalisation of multi-edge spinal groups, studied in [1, and extended Gupta-Sidki groups, introduced in [21]. Note that the class of multi-edge spinal groups includes all GGS-groups. With a certain amount of care we extend the main results in [1, 21, 25, 27] to groups in the larger class $\mathscr{C}$. The extended GuptaSidki groups, originally manufactured by Pervova as examples of just infinite

2010 Mathematics Subject Classification. Primary 20E08; Secondary 20E28, 20C07.

Key words and phrases. Tree automorphisms, branch groups, maximal subgroups, irreducible representations. 
branch groups without the congruence subgroup property, seem to have received little attention beyond [21. It is reassuring that results first obtained for multi-edge spinal groups carry over to these groups.

For convenience, we give now an abridged definition of the class $\mathscr{C}$ of generalised multi-edge spinal groups and illustrate the concept with simple examples. A more detailed discussion and the definitions of standard terms can be found in Section 2

A generalised multi-edge spinal group

$$
G=\left\langle\{a\} \cup\left\{b_{i}^{(j)} \mid 1 \leq j \leq p, 1 \leq i \leq r_{j}\right\}\right\rangle
$$

is an infinite subgroup of (a Sylow-pro- $p$ subgroup of) the profinite group $\operatorname{Aut}(T)$ that is generated by

- a rooted automorphism $a$ of order $p$ permuting cyclically the vertices $u_{1}, \ldots, u_{p}$ at the 1 st level of $T$, and

- families $\mathbf{b}^{(j)}=\left\{b_{1}^{(j)}, \ldots, b_{r_{j}}^{(j)}\right\}, j \in\{1, \ldots, p\}$, of directed automorphisms sharing a common directed path $P_{j}$ in $T$.

The paths $P_{1}, \ldots, P_{p}$ are required to be mutually disjoint. Without loss of generality we can demand that none of the generators are superfluous, hence $0 \leq r_{j} \leq p-1$ for all $j \in\{1, \ldots, p\}$. Since $G$ is infinite, there is at least one $j \in\{1, \ldots, p\}$ such that $r_{j} \neq 0$.

By construction such a generalised multi-edge spinal group is a finitely generated, residually-(finite $p$ ) infinite group. Regarded as a subgroup of $\operatorname{Aut}(T)$ it is fractal, and under additional assumptions, as we will see below, it is just infinite and branch.

Example 1.1. The extended Gupta-Sidki group, or EGS-group for short, with defining vector $\mathbf{e}=\left(e_{1}, \ldots, e_{p-1}\right) \in(\mathbb{Z} / p \mathbb{Z})^{p-1} \backslash\{\mathbf{0}\}$ is the group

$$
G=\langle a, b, c\rangle \leq \operatorname{Aut}(T),
$$

where the rooted automorphism $a$ permutes cyclically the $p$ vertices at the 1st level of $T$, whereas the two directed automorphisms $b, c$ belong to the 1st level stabiliser $\operatorname{Stab}_{G}(1)$ and satisfy the recursion relations

$$
b=\left(a^{e_{1}}, \ldots, a^{e_{p-1}}, b\right) \text { and } c=\left(c, a^{e_{1}}, \ldots, a^{e_{p-1}}\right) .
$$

Observe that each of the generators $a, b, c$ has order $p$. In 21, Pervova imposes two additional requirements: $\sum_{i=1}^{p-1} e_{i}=0$ and $\mathbf{e}$ is non-symmetric, i.e., $e_{i} \neq e_{p-i}$ for some $i \in\{1, \ldots, p-1\}$. The first condition is equivalent to $G$ being torsion; see [24, Theorem 2] and [28, Theorem 1]. Pervova shows under these assumptions: $G$ is just infinite and branch, but does not have the congruence subgroup property; see [21]. This is in contrast to the Grigorchuk group [22, Theorem 3.1] and branch GGS-groups [9, Theorem A].

Example 1.2. Given $r \in\{1, \ldots, p-1\}$ and a finite $r$-tuple $\mathbf{E}$ of $(\mathbb{Z} / p \mathbb{Z})$ linearly independent vectors

$$
\mathbf{e}_{i}=\left(e_{i, 1}, e_{i, 2}, \ldots, e_{i, p-1}\right) \in(\mathbb{Z} / p \mathbb{Z})^{p-1}, \quad i \in\{1, \ldots, r\},
$$


we recursively define directed automorphisms $b_{1}, \ldots, b_{r}$ via

$$
b_{i}=\left(a^{e_{i, 1}}, a^{e_{i, 2}}, \ldots, a^{e_{i, p-1}}, b_{i}\right), \quad i \in\{1, \ldots, r\} .
$$

The group $G=\left\langle a, b_{1}, \ldots, b_{r}\right\rangle \leq \operatorname{Aut}(T)$ is the multi-edge spinal group associated to the defining vectors $\mathbf{E}$. We observe that $\langle a\rangle \cong C_{p}$ and $\left\langle b_{1}, \ldots, b_{r}\right\rangle \cong$ $C_{p}^{r}$ are elementary abelian $p$-groups. These groups generalise GGS-groups, which correspond to the special case $r=1$. In [1] it was seen that the torsion multi-edge spinal groups are just infinite and branch. Moreover, generalising results of Pervova on GGS-groups, it was shown there that torsion multi-edge spinal groups do not contain maximal subgroups of infinite index. Equivalently, these groups do not contain proper dense subgroups with respect to the profinite topology.

Remark 1.3. We will have occasion to look at generalised Gupta-Sidki groups. By this we mean GGS-groups $G=\left\langle a, b_{1}\right\rangle$, i.e. $r=1$ in the notation above, with the extra property that $\left\{e_{1,1}, \ldots, e_{1, p-1}\right\}=\{1,2, \ldots, p-1\}$. This definition subsumes the generalised Gupta-Sidki group $\langle a, b\rangle$, studied in [27, where $b=\left(a, a^{2}, \ldots, a^{p-1}, b\right)$. For $p=3$ this group is the Gupta-Sidki 3-group.

We extend the results illustrated by the two examples as follows.

Theorem 1.4. Let $G$ be a generalised multi-edge spinal group, that is a group in the class $\mathscr{C}$ as described in (1.1).

(1) If every non-empty family $\mathbf{b}^{(j)}, j \in\{1, \ldots, p\}$, features at least one non-constant defining vector, then $G$ is regular branch over $\gamma_{3}(G)$.

Consequently, if $G$ is torsion then $G$ is just infinite and branch.

(2) If the group $G$ is torsion then $G$ does not contain any proper dense subgroups, with respect to the profinite topology. The same holds for groups commensurable to $G$.

(3) Suppose that the families $\mathbf{b}^{(1)}, \ldots, \mathbf{b}^{(p)}$ of directed generators of $G$ satisfy the additional conditions:

(i) every non-empty family $\mathbf{b}^{(j)}, j \in\{1, \ldots, p\}$, features at least one non-symmetric defining vector;

(ii) there are at least two directed automorphisms, from two distinct families, that have the same defining vector.

Then $G$ does not have the congruence subgroup property.

For comparison, we remark that Bondarenko [5] has shown that there exist finitely generated branch groups that possess maximal subgroups of infinite index. Bou-Rabee, Leemann and Nagnibeda [6] proceeded to investigate weakly maximal subgroups of branch groups, that is those that are maximal among the subgroups of infinite index. The Grigorchuk group, the GuptaSidki group and many other GGS-groups contain uncountably many nonparabolic weakly maximal subgroups. In Corollary 3.12 we observe that these results also apply to the branch groups in $\mathscr{C}$. 
Returning to the results about maximal subgroups, as explained in [1, 20] they relate to a conjecture of Passman [17, Conjecture 6.1] on the group algebra $F[G]$ of a finitely generated group $G$ over a field $F$ of characteristic $p$. The conjecture states that, if the Jacobson radical $\operatorname{Jac}(F[G])$ coincides with the augmentation ideal $\operatorname{Aug}(F[G])$, then $G$ is a finite p-group. In [17, Passman proved that if $\operatorname{Jac}(F[G])=\operatorname{Aug}(F[G])$ then $G$ is a $p$-group and every maximal subgroup of $G$ is normal of index $p$.

The torsion groups in $\mathscr{C}$, having the property that maximal subgroups are normal of index $p$, form a natural supply of potential counter-examples to Passman's conjecture. However, prominent examples such as the Grigorchuk group and the generalised Gupta-Sidki $p$-groups, do not satisfy the prerequisite $\operatorname{Jac}(F[G])=\operatorname{Aug}(F[G])$; see [2, 3, 25, 27]. In Section 6, we prove similar results for a larger class of groups in $\mathscr{C}$.

The following question of Bergman [16, Problem 17.17] was brought to our attention by A. Abdollahi: do there exist finitely generated infinite groups with finitely many maximal subgroups. As recorded in the Kourovka Notebook, an example of a 2-generated 2-group with 3 maximal subgroups can be extracted from [8, Sec. 7]. Due to Theorem 1.4(2) we obtain a large and more easily describable collection of finitely generated infinite groups with finitely many maximal subgroups.

Now a subgroup $G \leq \operatorname{Aut}(T)$ naturally acts on the boundary $\partial T$ of the tree $T$. The tree enveloping algebra $\mathfrak{A}_{G}$ of $G$ over the prime field $F=$ $\mathbb{Z} / p \mathbb{Z}$ is the image of $F[G]$ in the endomorphism algebra $\operatorname{End}(F\langle\langle\partial T\rangle\rangle)$ of the $F$-vector space on the basis $\partial T$. The image of $\operatorname{Aug}(F[G])$ in $\mathfrak{A}_{G}$ is denoted by $\operatorname{Aug}\left(\mathfrak{A}_{G}\right)$. Bartholdi [2, 3] extensively studied the tree enveloping algebra of the Grigorchuk group, over various fields. One of his key results is that the tree enveloping algebra of the Grigorchuk group over a field of characteristic 2 has a natural grading [2, Corollary 4.16]. This can be concluded from the recursive presentation of the tree enveloping algebra [2, Theorem 4.15]. It remains open, as to whether the tree enveloping algebra of the Gupta-Sidki groups, or of other GGS-groups, similarly admit a natural grading. We see from the following that it is desirable to close this gap in our knowledge.

Theorem 1.5. Let $G=\left\langle a, \mathbf{b}^{(1)}, \ldots, \mathbf{b}^{(p)}\right\rangle$ be a just infinite group in $\mathscr{C}$, and let $\mathfrak{A}_{G}$ be its tree enveloping algebra over $F=\mathbb{Z} / p \mathbb{Z}$.

If, either $G$ contains a generalised Gupta-Sidki group as a subgroup, or the induced augmentation ideal $\operatorname{Aug}\left(\mathfrak{A}_{G}\right)$ is a graded algebra with the elements $a-1$ and $b_{i}^{(j)}-1$ for $1 \leq j \leq p, 1 \leq i \leq r_{j}$ being homogeneous, then $G$ admits a faithful infinite-dimensional irreducible F-representation.

Remark 1.6. The proof of Theorem 1.5 reveals that the groups $G$ to which it applies satisfy $\operatorname{Jac}\left(\mathfrak{A}_{G}\right) \neq \operatorname{Aug}\left(\mathfrak{A}_{G}\right)$. This implies that $\operatorname{Jac}(F[G]) \neq$ $\operatorname{Aug}(F[G])$, and hence, even though it may be torsion, $G$ cannot be a counter-example to Passman's Conjecture. 
Passman and Temple showed in [19] that for the Gupta-Sidki $p$-group $\mathrm{GS}_{p}$ and $E$ an algebraically closed field of characteristic $p$, if $E\left[\mathrm{GS}_{p}\right]$ has a non-trivial irreducible module, then $E\left[\mathrm{GS}_{p}\right]$ has infinitely many irreducible modules. We extend their result to branch groups in $\mathscr{C}$.

Theorem 1.7. Let $G$ be a branch group in $\mathscr{C}$ and let $E$ be an algebraically closed field of characteristic $p$. If $E[G]$ has a non-trivial irreducible module, then $E[G]$ has infinitely many irreducible modules.

\section{Preliminaries}

Let $T$ be the regular $p$-adic rooted tree, meaning all vertices have the same out-degree $p$. Using the alphabet $X=\{1,2, \ldots, p\}$, the vertices $u_{\omega}$ of $T$ are labelled bijectively by elements $\omega$ of the free monoid $\bar{X}$ in the following natural way. The root of $T$ is labelled by the empty word $\varnothing$, and for each word $\omega \in \bar{X}$ and letter $x \in X$ there is an edge connecting $u_{\omega}$ to $u_{\omega x}$. More generally, we say that $u_{\omega}$ precedes $u_{\lambda}$, or equivalently that $u_{\lambda}$ succeeds $u_{\omega}$, whenever $\omega$ is a prefix of $\lambda$.

There is a natural length function on $\bar{X}$. The words $\omega$ of length $|\omega|=n$, representing vertices $u_{\omega}$ that are at distance $n$ from the root, are the $n$th level vertices and constitute the nth layer of the tree; the boundary $\partial T$, whose elements correspond naturally to infinite rooted paths, is in one-toone correspondence with the $p$-adic integers.

Denote by $T_{u}$ the full rooted subtree of $T$ that has its root at a vertex $u$ and includes all vertices succeeding $u$. For any two vertices $u=u_{\omega}$ and $v=u_{\lambda}$, the map $u_{\omega \tau} \mapsto u_{\lambda \tau}$, induced by replacing the prefix $\omega$ by $\lambda$, yields an isomorphism between the subtrees $T_{u}$ and $T_{v}$. We write $T_{n}$ to denote the subtree rooted at a generic vertex of level $n$.

Every automorphism of $T$ fixes the root and the orbits of $\operatorname{Aut}(T)$ on the vertices of the tree $T$ are precisely its layers. For $f \in \operatorname{Aut}(T)$, the image of a vertex $u$ under $f$ is denoted by $u^{f}$. Observe that $f$ induces a faithful action on the monoid $\bar{X}$ such that $\left(u_{\omega}\right)^{f}=u_{\omega f}$. For $\omega \in \bar{X}$ and $x \in X$ we have $(\omega x)^{f}=\omega^{f} x^{\prime}$ where $x^{\prime} \in X$ is uniquely determined by $\omega$ and $f$. This induces a permutation $f(\omega)$ of $X$ so that

$$
(\omega x)^{f}=\omega^{f} x^{f(\omega)}, \quad \text { and consequently } \quad\left(u_{\omega x}\right)^{f}=u_{\omega^{f} x^{f(\omega)}} .
$$

The automorphism $f$ is rooted if $f(\omega)=1$ for $\omega \neq \varnothing$. It is directed, with directing path $\ell \in \partial T$, if the support $\{\omega \mid f(\omega) \neq 1\}$ of its labelling is infinite and marks only vertices at distance 1 from the set of vertices corresponding to the path $\ell$.

2.1. Subgroups of $\operatorname{Aut}(T)$. Let $G$ be a subgroup of Aut $(T)$ acting spherically transitively, that is, transitively on every layer of $T$. The vertex stabiliser $\operatorname{Stab}_{G}(u)$ is the subgroup consisting of elements in $G$ that fix the vertex $u$. For $n \in \mathbb{N}$, the $n$th level stabiliser $\operatorname{Stab}_{G}(n)=\bigcap_{|\omega|=n} \operatorname{Stab}_{G}\left(u_{\omega}\right)$ is the subgroup consisting of automorphisms that fix all vertices at level $n$. 
Denoting by $T_{[n]}$ the finite subtree of $T$ on vertices up to level $n$, we see that $\operatorname{Stab}_{G}(n)$ is equal to the kernel of the induced action of $G$ on $T_{[n]}$.

The full automorphism group $\operatorname{Aut}(T)$ is a profinite group:

$$
\operatorname{Aut}(T)=\lim _{n \rightarrow \infty} \operatorname{Aut}\left(T_{[n]}\right)
$$

The topology of $\operatorname{Aut}(T)$ is defined by the open subgroups $\operatorname{Stab}_{\operatorname{Aut}(T)}(n)$, $n \in \mathbb{N}$. The subgroup $G$ of $\operatorname{Aut}(T)$ has the congruence subgroup property if for every subgroup $H$ of finite index in $G$, there exists some $n$ such that $\operatorname{Stab}_{G}(n) \subseteq H$. For branch groups, having the congruence subgroup property is independent of the choice of tree and action; see [12. In fact, in most of the cases that we consider, there is essentially a unique tree and action associated to the group; cf. Corollary 3.8.

Each $g \in \operatorname{Stab}_{\operatorname{Aut}(T)}(n)$ can be described completely in terms of its restrictions to the subtrees rooted at vertices at level $n$. Indeed, there is a natural isomorphism

$$
\psi_{n}: \operatorname{Stab}_{\operatorname{Aut}(T)}(n) \rightarrow \prod_{|\omega|=n} \operatorname{Aut}\left(T_{u_{\omega}}\right) \cong \operatorname{Aut}(T) \times p^{n} . \times \operatorname{Aut}(T) .
$$

We write $U_{u}^{G}$ for the restriction of the vertex stabiliser $\operatorname{Stab}_{G}(u)$ to the subtree $T_{u}$ rooted at a vertex $u$. Since $G$ acts spherically transitively, the vertex stabilisers at every level are conjugate under $G$. The common isomorphism type of the restriction of the $n$th level vertex stabilisers is the $n t h$ upper companion group $U_{n}^{G}$ of $G$. The group $G$ is fractal if every upper companion group $U_{n}^{G}$ coincides with the group $G$, after the natural identification of subtrees.

The rigid vertex stabiliser of $u$ in $G$ is the subgroup $\operatorname{Rstab}_{G}(u)$ consisting of all automorphisms in $G$ that fix all vertices $v$ of $T$ not succeeding $u$. The rigid $n$th level stabiliser is the product

$$
\operatorname{Rstab}_{G}(n)=\prod_{|\omega|=n} \operatorname{Rstab}_{G}\left(u_{\omega}\right) \unlhd G
$$

of the rigid vertex stabilisers of the vertices at level $n$. The rigid vertex stabilisers at each level are conjugate under $G$ and the common isomorphism type $L_{n}^{G}$ of the $n$th level rigid vertex stabilisers is called the $n$th lower companion group of $G$.

We recall that the spherically transitive group $G$ is a branch group, if $\operatorname{Rstab}_{G}(n)$ has finite index in $G$ for every $n \in \mathbb{N}$. For more detailed algebraic and geometric characterisations see [13]. If, in addition, $G$ is fractal and $1 \neq K \leq \operatorname{Stab}_{G}(1)$ with $K \times \ldots \times K \subseteq \psi_{1}(K)$ and $|G: K|<\infty$, then $G$ is said to be regular branch over $K$. Lastly we note that an infinite group $G$ is just infinite if all its proper quotients are finite.

2.2. The collection $\mathscr{C}$ of generalised multi-edge spinal groups. For $j \in\{1, \ldots, p\}$ let $r_{j} \in\{0,1, \ldots, p-1\}$, with $r_{j} \neq 0$ for at least one index $j$, and fix the numerical datum $\mathbf{E}=\left(\mathbf{E}^{(1)}, \ldots, \mathbf{E}^{(p)}\right)$, where each 
$\mathbf{E}^{(j)}=\left(\mathbf{e}_{1}^{(j)}, \ldots, \mathbf{e}_{r_{j}}^{(j)}\right)$ is an $r_{j}$-tuple of $(\mathbb{Z} / p \mathbb{Z})$-linearly independent vectors

$$
\mathbf{e}_{i}^{(j)}=\left(e_{i, 1}^{(j)}, \ldots, e_{i, p-1}^{(j)}\right) \in(\mathbb{Z} / p \mathbb{Z})^{p-1}, \quad i \in\left\{1, \ldots, r_{j}\right\} .
$$

By $a$ we denote the rooted automorphism, corresponding to the $p$-cycle $(12 \ldots p) \in \operatorname{Sym}(p)$, that cyclically permutes the vertices at the first level of $T$. Observe that

$$
S=\{f \in \operatorname{Aut}(T) \mid \forall \omega \in \bar{X}: f(\omega) \in\langle a\rangle\} \cong \lim _{n \in \mathbb{N}} C_{p} \prec \ldots \curlywedge C_{p} \prec C_{p},
$$

the inverse limit of $n$-fold iterated wreath products of $C_{p}$, forms a Sylowpro- $p$ subgroup of Aut $(T)$. The generalised multi-edge spinal group in standard form associated to the datum $\mathbf{E}$ is the group

$$
\begin{aligned}
G=G_{\mathbf{E}} & =\left\langle a, \mathbf{b}^{(1)}, \ldots, \mathbf{b}^{(p)}\right\rangle \\
& =\left\langle\{a\} \cup\left\{b_{i}^{(j)} \mid 1 \leq j \leq p, 1 \leq i \leq r_{j}\right\}\right\rangle \leq S,
\end{aligned}
$$

where, for each $j \in\{1, \ldots, p\}$, the generator family $\mathbf{b}^{(j)}=\left\{b_{1}^{(j)}, \ldots, b_{r_{j}}^{(j)}\right\}$

consists of commuting directed automorphisms $b_{i}^{(j)} \in \operatorname{Stab}_{G}(1)$ along the directed path

$$
(\varnothing,(p-j+1),(p-j+1)(p-j+1), \ldots) \in \partial T
$$

that satisfy the recursive relations

$$
\psi_{1}\left(b_{i}^{(j)}\right)=\left(a^{e_{i, j}^{(j)}}, \ldots, a^{e_{i, p-1}^{(j)}}, b_{i}^{(j)}, a^{e_{i, 1}^{(j)}}, \ldots, a^{e_{i, j-1}^{(j)}}\right) ;
$$

sometimes $\mathbf{e}_{i}^{(j)}$ is called the defining vector of $b_{i}^{(j)}$. For each $j \in\{1, \ldots, p\}$ with $r_{j} \neq 0$ the subgroup $\left\langle a, \mathbf{b}^{(j)}\right\rangle=\left\langle a, b_{1}^{(j)}, \ldots, b_{r_{j}}^{(j)}\right\rangle$ of $G$ is a multi-edge spinal group; compare Example 1.2 .

Observe that the directing paths for the generator families $\mathbf{b}^{(1)}, \ldots, \mathbf{b}^{(p)}$, i.e., the paths

$$
(\varnothing, p, p p, \ldots), \quad(\varnothing,(p-1),(p-1)(p-1), \ldots), \quad \ldots, \quad(\varnothing, 1,11, \ldots),
$$

are pairwise distinct. We arrive at the notion of a generalised multi-edge spinal group, given in abridged form in Section 1, by considering subgroups of $\operatorname{Aut}(T)$ that are conjugate to a generalised multi-edge spinal group in standard form and declaring $\mathscr{C}$ to be the class of all such groups. Whenever they are branch, there is, in fact, a unique branch action associated to these groups; see Corollary 3.8 .

\section{First PROPERTIES OF GENERALISED MULTI-EDGE SPINAL GROUPS}

3.1. Basic properties. Here we include basic results concerning generalised multi-edge spinal groups, i.e., groups in the class $\mathscr{C}$. Directly from the definition we deduce that each group in $\mathscr{C}$ is infinite, fractal and acts spherically transitively on $T$. 
Lemma 3.1. Let $G=\left\langle a, \mathbf{b}^{(1)}, \ldots, \mathbf{b}^{(p)}\right\rangle \in \mathscr{C}$ be in standard form, and let $k \in\{1, \ldots, p\}$ with $r_{k} \neq 0$. There exists an automorphism $f \in \operatorname{Aut}(T)$ of the form $f=f_{0} f_{1}=f_{1} f_{0}$, where $f_{0}$ is a rooted automorphism corresponding to a permutation $\pi \in \operatorname{Sym}(p)$ with $(p-k+1) \pi=p-k+1$ and $f_{1} \in \operatorname{Stab}_{G}(1)$ with $\psi_{1}\left(f_{1}\right)=(f, \ldots, f)$, such that $G^{f}=\left\langle a, \tilde{\mathbf{b}}^{(1)}, \ldots, \tilde{\mathbf{b}}^{(p)}\right\rangle \in \mathscr{C}$ is again in standard form and satisfies $\psi_{1}\left(\tilde{b}_{1}^{(k)}\right)=\left(a^{\tilde{e}_{1, k}^{(k)}}, \ldots, a^{\tilde{e}_{1, p-1}^{(k)}}, \tilde{b}_{1}^{(k)}, a, a^{\tilde{e}_{1,2}^{(k)}}, \ldots, a^{\tilde{e}_{1, k-1}^{(k)}}\right)$. Proof. In essence, we may use the same proof as that of [1, Lemma 3.3].

Next we recall and adapt a reduction lemma from [1] to the new situation.

Lemma 3.2 ([1, Lemma 3.4]). Let $G=\left\langle a, \mathbf{b}^{(1)}, \ldots, \mathbf{b}^{(p)}\right\rangle \in \mathscr{C}$ be in standard form, and let $k \in\{1, \ldots, p\}$ with $r=r_{k} \neq 0$. Then there exists a group $\tilde{G}=\left\langle a, \tilde{\mathbf{b}}^{(1)}, \ldots, \tilde{\mathbf{b}}^{(p)}\right\rangle \in \mathscr{C}$ in standard form associated to the datum $\tilde{\mathbf{E}}=$ $\left(\tilde{\mathbf{E}}^{(1)}, \ldots, \tilde{\mathbf{E}}^{(p)}\right)$, with $\tilde{\mathbf{E}}^{(j)}=\left(\tilde{\mathbf{e}}_{1}^{(j)}, \ldots, \tilde{\mathbf{e}}_{r_{j}}^{(j)}\right)$ supplying defining vectors for the directed automorphisms $\tilde{\mathbf{b}}^{(j)}=\left(\tilde{b}_{1}^{(j)}, \ldots, \tilde{b}_{r_{j}}^{(j)}\right)$, such that $\tilde{G}$ is conjugate to $G$ by an element $f \in \operatorname{Aut}(T)$ as in Lemma 3.1 and the following holds:

(1) $\tilde{e}_{i, 1}^{(k)}=1$ in $\mathbb{Z} / p \mathbb{Z}$ for each $i \in\{1, \ldots, r\}$;

(2) if $r=2$ and $p=3$, then $\tilde{\mathbf{e}}_{1}^{(k)}=(1,0), \tilde{\mathbf{e}}_{2}^{(k)}=(1,1)$;

(3) if $r=2$ and $p>3$, then either

(a) for each $i \in\{1,2\}$ there exists $m \in\{2, \ldots, p-2\}$ such that $\tilde{e}_{i, m-1}^{(k)} \tilde{e}_{i, m+1}^{(k)} \neq\left(\tilde{e}_{i, m}^{(k)}\right)^{2}$ in $\mathbb{Z} / p \mathbb{Z}$, or

(b) $\tilde{\mathbf{e}}_{1}^{(k)}=(1,0, \ldots, 0,0), \tilde{\mathbf{e}}_{2}^{(k)}=(1,0, \ldots, 0,1)$;

(4) if $r \geq 3$ then for each $i \in\{1, \ldots, r\}$ there exists $m \in\{2, \ldots, p-2\}$ such that $\tilde{e}_{i, m-1}^{(k)} \tilde{e}_{i, m+1}^{(k)} \neq\left(\tilde{e}_{i, m}^{(k)}\right)^{2}$ in $\mathbb{Z} / p \mathbb{Z}$.

As in [1, Section 3], we identify some 'exceptional' groups to be excluded from some of our results: let $\mathscr{C}_{\text {reg }}$ be the class of groups that are conjugate in $\operatorname{Aut}(T)$ to a group $\left\langle a, \mathbf{b}^{(1)}, \ldots, \mathbf{b}^{(p)}\right\rangle \in \mathscr{C}$ in standard form such that every non-empty generator family $\mathbf{b}^{(j)}, j \in\{1, \ldots, p\}$, features at least one non-constant defining vector $\mathbf{e}_{i}^{(j)} \notin\{(\alpha, \ldots, \alpha) \mid \alpha \in \mathbb{Z} / p \mathbb{Z}\}$ with $1 \leq i \leq r_{j}$.

Proposition 3.3. Let $G=\left\langle a, \mathbf{b}^{(1)}, \ldots, \mathbf{b}^{(p)}\right\rangle \in \mathscr{C}_{\text {reg }}$ be in standard form. Then

In particular,

$$
\psi_{1}\left(\gamma_{3}\left(\operatorname{Stab}_{G}(1)\right)\right)=\gamma_{3}(G) \times \stackrel{p}{.} \times \gamma_{3}(G) .
$$

$$
\gamma_{3}(G) \times \stackrel{p}{.} \times \gamma_{3}(G) \subseteq \psi_{1}\left(\gamma_{3}(G)\right),
$$

and $G$ is regular branch over $\gamma_{3}(G)$.

Proof. By spherical transitivity, it suffices to show that

$$
\gamma_{3}(G) \times 1 \times \ldots \times 1 \subseteq \psi_{1}\left(\gamma_{3}\left(\operatorname{Stab}_{G}(1)\right)\right) .
$$

Observe that $\gamma_{3}(G)$ is generated as a normal subgroup by commutators $\left[g_{1}, g_{2}, g_{3}\right]$ of elements $g_{1}, g_{2}, g_{3}$ ranging over the generating set $\{a\} \cup\left\{b_{i}^{(j)} \mid\right.$ $\left.1 \leq j \leq p, 1 \leq i \leq r_{j}\right\}$. For each $j \in\{1, \ldots, p\}$ with $r_{j} \neq 0$, the subgroup 
$G_{j}=\left\langle a, \mathbf{b}^{(j)}\right\rangle \leq G$ is a multi-edge spinal group, and [1, Proposition 3.5] shows that

$$
\gamma_{3}\left(G_{j}\right) \times \stackrel{p}{.} \times \gamma_{3}\left(G_{j}\right) \subseteq \psi_{1}\left(\gamma_{3}\left(\operatorname{Stab}_{G_{j}}(1)\right)\right) \subseteq \psi_{1}\left(\gamma_{3}\left(\operatorname{Stab}_{G}(1)\right)\right) .
$$

Hence, it suffices to prove, for $k, l, m \in\{1, \ldots, p\}$ with $k \neq l$ and any given $c_{j} \in\left\{b_{1}^{(j)}, \ldots, b_{r_{j}}^{(j)}\right\}, j \in\{k, l, m\}$, the elements

$$
\left(\left[a, c_{k}, c_{l}\right], 1, \ldots, 1\right), \quad\left(\left[c_{k}, c_{l}, a\right], 1, \ldots, 1\right), \quad\left(\left[c_{k}, c_{l}, c_{m}\right], 1, \ldots, 1\right)
$$

are contained in $\psi_{1}\left(\gamma_{3}\left(\operatorname{Stab}_{G}(1)\right)\right)$.

First, we observe that

$$
\left(\left[c_{k}, c_{l}, c_{m}\right], 1, \ldots, 1\right)=\psi_{1}\left(\left[c_{k}^{a^{k}}, c_{l}^{a^{l}}, c_{m}^{a^{m}}\right]\right) \in \psi_{1}\left(\gamma_{3}\left(\operatorname{Stab}_{G}(1)\right)\right) .
$$

Similarly, as $\psi_{1}\left(\left[c_{k}^{a^{k}}, c_{l}^{a^{l}}\right]\right)=\left(\left[c_{k}, c_{l}\right], 1, \ldots, 1\right)$, we can take $d \in \operatorname{Stab}_{G}(1)$ such that $\psi_{1}(d)=(a, *, \ldots, *)$, where the symbols $*$ denote unspecified elements, to deduce that

$$
\left(\left[c_{k}, c_{l}, a\right], 1, \ldots, 1\right)=\psi_{1}\left(\left[c_{k}^{a^{k}}, c_{l}^{a^{l}}, d\right]\right) \in \psi_{1}\left(\gamma_{3}\left(\operatorname{Stab}_{G}(1)\right)\right) .
$$

It remains to deal with the first type of commutator $\left(\left[a, c_{k}, c_{l}\right], 1, \ldots, 1\right)$ listed in (3.1). Working with a fixed $k \in\{1, \ldots, p\}$, but still allowing for modifications of the specific generators, we are free to conjugate by an element $f \in \operatorname{Aut}(T)$ as in Lemma 3.1, and without loss of generality we may assume that the defining vectors $\mathbf{E}^{(k)}$ of the generators $\mathbf{b}^{(k)}$ have the form of $\tilde{\mathbf{E}}^{(k)}$ described in Lemma 3.2 .

Let us specify $c_{k}=b_{i}^{(k)}$ and $c_{l}=b_{j}^{(l)}$, where $i \in\left\{1, \ldots, r_{k}\right\}$ and $j \in$ $\left\{1, \ldots, r_{l}\right\}$. Moreover, as we have already seen that $\gamma_{3}\left(G_{k}\right) \times 1 \times \ldots \times 1 \subseteq$ $\psi_{1}\left(\gamma_{3}\left(\operatorname{Stab}_{G}(1)\right)\right)$, it suffices to prove that there exists $x \in G_{k}$ such that

$$
\begin{aligned}
\left(\left[a, b_{i}^{(k)}, b_{j}^{(l)}\right]\left[a, b_{i}^{(k)}, x\right]^{b_{j}^{(l)}},\right. & 1, \ldots, 1) \\
& =\left(\left[a, b_{i}^{(k)}, x b_{j}^{(l)}\right], 1, \ldots, 1\right) \in \psi_{1}\left(\gamma_{3}\left(\operatorname{Stab}_{G}(1)\right)\right) .
\end{aligned}
$$

Arguing similar to [1, Proof of Proposition 3.5], we distinguish between three situations.

Case 1: $r_{k}=1$, and thus $i=1$. Observing that

$$
h_{j}=\left(\left(b_{1}^{(k)}\right)^{a^{k-2}}\right)^{-s}\left(b_{j}^{(l)}\right)^{a^{l}}=\left(x b_{j}^{(l)}, *, \ldots, *, 1\right)
$$

for $s=e_{j, p-1}^{(l)}$ and $x \in G_{k}$, we obtain

$$
\left(\left[a, b_{1}^{(k)}, x b_{j}^{(l)}\right], 1, \ldots, 1\right)=\psi_{1}\left(\left[\left(b_{1}^{(k)}\right)^{a^{k-1}},\left(b_{1}^{(k)}\right)^{a^{k}}, h_{j}\right]\right) \in \psi_{1}\left(\gamma_{3}\left(\operatorname{Stab}_{G}(1)\right)\right) .
$$

Case 2: $r_{k}>1$ and $\left(r_{k}, p\right) \neq(2,3)$. By properties (3) and (4) of Lemma 3.2 there exists $m \in\{2, \ldots, p-2\}$ such that $e_{i, m-1}^{(k)} e_{i, m+1}^{(k)} \neq\left(e_{i, m}^{(k)}\right)^{2}$; apart from an exceptional case which only occurs for $r_{k}=2$ to be dealt with below. We set

$$
g_{i, m}=\left(\left(b_{i}^{(k)}\right)^{a^{k-m}}\right)^{e_{i, m}^{(k)}}\left(\left(b_{i}^{(k)}\right)^{a^{k-m-1}}\right)^{-e_{i, m-1}^{(k)}}
$$


which gives

$$
\psi_{1}\left(g_{i, m}\right)=\left(a^{\left.\left(e_{i, m}^{(k)}\right)^{2}-e_{i, m-1}^{(k)} e_{i, m+1}^{(k)}, *, \ldots, *, 1\right)},\right.
$$

where, in this case, the unspecified elements $*$ lie in $\left\langle a, b_{i}^{(k)}\right\rangle \leq G_{k}$. Since the first entry is non-trivial, there is a power $g_{i}$ of $g_{i, m}$ such that

$$
\psi_{1}\left(g_{i}\right)=(a, y, *, \ldots, *, 1), \quad \text { where } y \in G_{k} .
$$

Motivated by

$$
\psi_{1}\left(\left(b_{j}^{(l)}\right)^{a^{l}}\right)=\left(b_{j}^{(l)}, *, \ldots, *, a^{s}\right), \quad \text { where } s=e_{j, p-1}^{(l)},
$$

and

$$
\psi_{1}\left(\left(g_{i}^{a^{-1}}\right)^{-s}\right)=\left(x, *, \ldots, *, 1, a^{-s}\right) \quad \text { where } x=y^{-s},
$$

we define $h_{j}=\left(g_{i}^{a^{-1}}\right)^{-s}\left(b_{j}^{(l)}\right)^{a^{l}}$ so that

$$
\psi_{1}\left(h_{j}\right)=\left(x b_{j}^{(l)}, *, \ldots, *, 1\right) .
$$

As $e_{i, 1}^{(k)}=1$, it follows that

$$
\left(\left[a, b_{i}^{(k)}, x b_{j}^{(l)}\right], 1, \ldots, 1\right)=\psi_{1}\left(\left[\left(b_{i}^{(k)}\right)^{a^{k-1}},\left(b_{i}^{(k)}\right)^{a^{k}}, h_{j}\right]\right) \in \psi_{1}\left(\gamma_{3}\left(\operatorname{Stab}_{G}(1)\right)\right)
$$

and so we are done.

It remains to deal with the exceptional case which occurs only for $r_{k}=2$, and hence $p>3$. According to property (3b) of Lemma 3.2 ,

$$
\mathbf{e}_{1}^{(k)}=(1,0, \ldots, 0), \quad \mathbf{e}_{2}^{(k)}=(1,0, \ldots, 0,1),
$$

therefore

$$
\psi_{1}\left(b_{1}^{(k)}\right)^{a^{k-1}}=\left(a, 1, \ldots, 1, b_{1}^{(k)}\right), \quad \psi_{1}\left(b_{2}^{(k)}\right)^{a^{k-1}}=\left(a, 1, \ldots, 1, a, b_{2}^{(k)}\right) .
$$

In order to show that $\left(\left[a, b_{1}^{(k)}, b_{j}^{(l)}\right], 1, \ldots, 1\right)$ and $\left(\left[a, b_{2}^{(k)}, b_{j}^{(l)}\right], 1, \ldots, 1\right)$ are contained in $\psi_{1}\left(\gamma_{3}\left(\operatorname{Stab}_{G}(1)\right)\right)$, it suffices to replace $g_{i}$ in the generic argument given above by $\left(b_{2}^{(k)}\right)^{a^{k+1}}$ in both cases.

In fact, for the first element we can also argue directly as follows: from the relation $\psi_{1}\left(\left[\left(b_{1}^{(k)}\right)^{a^{k-1}},\left(b_{1}^{(k)}\right)^{a^{k}}\right]\right)=\left(\left[a, b_{1}^{(k)}\right], 1, \ldots, 1\right)$ we deduce

$$
\begin{aligned}
\left(\left[a, b_{1}^{(k)}, b_{j}^{(l)}\right], 1, \ldots, 1\right)=\psi_{1}\left(\left[\left(b_{1}^{(k)}\right)^{a^{k-1}},\left(b_{1}^{(k)}\right)^{a^{k}},\left(b_{j}^{(l)}\right)^{a^{l}}\right]\right) & \\
\in & \psi_{1}\left(\gamma_{3}\left(\operatorname{Stab}_{G}(1)\right)\right) .
\end{aligned}
$$

Case 3: $\left(r_{k}, p\right)=(2,3)$. By property (2) of Lemma 3.2, we may assume that

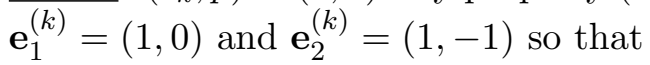

$$
\psi_{1}\left(\left(b_{1}^{(k)}\right)^{a^{k-1}}\right)=\left(a, 1, b_{1}^{(k)}\right) \quad \text { and } \quad \psi_{1}\left(\left(b_{2}^{(k)}\right)^{a^{k-1}}\right)=\left(a, a^{-1}, b_{2}^{(k)}\right) .
$$


Setting $h=\left(\left(b_{1}^{(k)}\right)^{a^{k-2}}\right)^{-s}\left(b_{j}^{(l)}\right)^{a^{l}}$ for $s=e_{j, p-1}^{(l)}$, we obtain $\psi_{1}(h)=\left(b_{j}^{(l)}, *, 1\right)$ and

$$
\begin{aligned}
& \left(\left[a, b_{1}^{(k)}, b_{j}^{(l)}\right], 1,1\right)=\psi_{1}\left(\left[\left(b_{1}^{(k)}\right)^{a^{k-1}},\left(b_{1}^{(k)}\right)^{a^{k}},\left(b_{j}^{(l)}\right)^{a^{l}}\right]\right), \\
& \left(\left[a, b_{2}^{(k)}, b_{j}^{(l)}\right], 1,1\right)=\psi_{1}\left(\left[\left(b_{1}^{(k)}\right)^{a^{k-1}},\left(b_{2}^{(k)}\right)^{a^{k}}, h\right]\right)
\end{aligned}
$$

so that both elements lie in $\psi_{1}\left(\gamma_{3}\left(\operatorname{Stab}_{G}(1)\right)\right)$.

Next we record the following result regarding the derived subgroup $G^{\prime}$ of $G$, based on the extra assumption that there are sufficiently many nonsymmetric defining vectors.

Proposition 3.4. Let $G=\left\langle a, \mathbf{b}^{(1)}, \ldots, \mathbf{b}^{(p)}\right\rangle \in \mathscr{C}$ be in standard form and such that every non-empty family $\mathbf{b}^{(j)}, j \in\{1, \ldots, p\}$, features at least one non-symmetric defining vector. Then

$$
\psi_{1}\left(\operatorname{Stab}_{G}(1)^{\prime}\right)=G^{\prime} \times \stackrel{p}{.} \times G^{\prime} .
$$

In particular, $G^{\prime} \times \stackrel{p}{.} \times G^{\prime} \subseteq \psi_{1}\left(G^{\prime}\right)$, and $G$ is regular branch over $G^{\prime}$.

Proof. By spherical transitivity, it suffices to show that

$$
G^{\prime} \times 1 \times \ldots \times 1 \subseteq \psi_{1}\left(\operatorname{Stab}_{G}(1)^{\prime}\right) .
$$

Observe that $G^{\prime}$ is generated as a normal subgroup by commutators $\left[g_{1}, g_{2}\right]$, where $g_{1}, g_{2}$ range over the generating set $\{a\} \cup\left\{b_{i}^{(j)} \mid 1 \leq j \leq p, 1 \leq i \leq r_{j}\right\}$.

For each $j \in\{1, \ldots, p\}$ with $r_{j} \neq 0$, we consider the associated multi-edge spinal group $G_{j}=\left\langle a, \mathbf{b}^{(j)}\right\rangle$. Without loss of generality we may assume that all defining vectors for the family $\mathbf{b}^{(j)}$ are non-symmetric. The $r_{j}$ subgroups

$$
H_{j}(1)=\left\langle a, b_{1}^{(j)}\right\rangle, \quad \cdots \quad, \quad H_{j}\left(r_{j}\right)=\left\langle a, b_{r_{j}}^{(j)}\right\rangle
$$

of $G_{j}$ are GGS-groups and satisfy the corresponding statement to our claim; see [10, Lemma 3.4]. Consequently,

$$
\left(\left[a, b_{i}^{(j)}\right], 1, \ldots, 1\right) \in \psi_{1}\left(\operatorname{Stab}_{H_{j}(i)}(1)^{\prime}\right) \subseteq \psi_{1}\left(\operatorname{Stab}_{G}(1)^{\prime}\right) \quad \text { for } 1 \leq i \leq r_{j} .
$$

Hence it suffices to observe that, for $k, l \in\{1, \ldots, p\}$ with $k \neq l$ and any given $c_{j} \in\left\{b_{1}^{(j)}, \ldots, b_{r_{j}}^{(j)}\right\}, j \in\{k, l\}$, the element $\left(\left[c_{k}, c_{l}\right], 1, \ldots, 1\right)=$ $\psi_{1}\left(\left[c_{k}^{a^{k}}, c_{l}^{a^{l}}\right]\right)$ is contained in $\psi_{1}\left(\operatorname{Stab}_{G}(1)^{\prime}\right)$.

Corollary 3.5. The groups in $\mathscr{C}_{\text {reg }}$ are branch, and the torsion groups in $\mathscr{C}$ are just infinite.

Proof. From Proposition 3.3, it follows similar to [1, Propositions 3.6 and 3.7] that the groups in $\mathscr{C}_{\text {reg }}$ are branch.

As indicated in Example 1.1. torsion groups in $\mathscr{C}$ are already in $\mathscr{C}_{\text {reg; }}$; see [24, Theorem 2] and [28, Theorem 1]. Finally, finitely generated torsion branch groups are just infinite; see [13, Section 7]. 
Hence we have established part (1) of Theorem 1.4 .

We end this section by proving that the branch groups in $\mathscr{C}$ have an essentially unique 'branch action'. For vertices $u, v$ of $T$ we write $u \leq v$ if $u$ precedes $v$, and $u<v$ for $u \leq v$, but $u \neq v$. In [14, Grigorchuk and Wilson (using a different notational convention) introduced the following condition on a branch group $G$ acting on a regular $p$-adic tree:

$(\dagger)$ Whenever $u, u^{\prime}, v$ are vertices of $T$ such that $u, u^{\prime}$ are incomparable and $u<v$, there exists $g \in G$ such that $\left(u^{\prime}\right)^{g}=u^{\prime}$, but $v^{g} \neq v$.

With this we state [14, Theorem 1], for branch groups acting on regular $p$-adic rooted trees.

Theorem 3.6 (Grigorchuk, Wilson). Let $G$ be a branch group acting on a regular $p$-adic rooted tree $T$ and suppose that $(\dagger)$ holds. Let $T^{\prime}$ be any other spherically homogeneous rooted tree on which $G$ acts as a branch group. Then there is a $G$-equivariant isomorphism from $T^{\prime}$ to a tree obtained from $T$ by deletion of layers.

This motivates us to prove the following result on GGS-groups.

Proposition 3.7. Let $G=\langle a, b\rangle$ be a GGS-group, acting on a regular $p$ adic tree $T$ for $p \geq 5$. Suppose further that the defining vector $\left(e_{1}, \ldots, e_{p-1}\right)$ of the directed automorphism $b$ is non-constant and that all its entries are non-zero. Then $G$ satisfies condition $(\dagger)$.

Proof. Let $u, u^{\prime}$ be incomparable vertices of $T$. We denote by $\bar{u}, \bar{u}^{\prime}$ the first level vertices satisfying $\bar{u} \leq u$ and $\bar{u}^{\prime} \leq u^{\prime}$. Suppose that $\bar{u}=u_{i}$ and $\bar{u}^{\prime}=u_{j}$ for labels $i, j \in\{1, \ldots, p\}$. Applying conjugation by a suitable power of $a$, if necessary, we may assume without loss of generality that $i<j$. Replacing $b$ by a suitable power of itself, if necessary, we may further assume that $e_{1}=1$.

Case 1: There exists $m \neq 1$ such that

$$
\psi_{1}(b)=\left(a, a^{m}, a^{m^{2}}, \ldots, a^{m^{p-2}}, b\right) .
$$

This implies

$$
\psi_{1}\left(b\left(b^{-m}\right)^{a}\right)=\left(a b^{-m}, 1, \ldots, 1, b a^{-1}\right) .
$$

First suppose that $(i, j) \neq(1, p)$. Then the element $g=\left(b\left(b^{-m}\right)^{a}\right)^{a^{i-1}} \in$ $\operatorname{Stab}_{G}(1)$ has the form

$$
\psi_{1}(g)=(1, \ldots, 1, b a^{-1}, \underbrace{a b^{-m}}_{i^{\text {th }} \text { entry }}, 1, \ldots, 1) .
$$

Consequently, $\left(u^{\prime}\right)^{g}=u^{\prime}$, but no vertex $v$ with $v>\bar{u}$ is fixed by $g$ due to the factor $a$ in the $i$ th entry of $\psi_{1}(g)$. Therefore condition $(\dagger)$ is satisfied.

In the remaining case $(i, j)=(1, p)$, we instead utilise $g=\left(b\left(b^{-m}\right)^{a}\right)^{a}$ where

$$
\psi_{1}(g)=\left(b a^{-1}, a b^{-m}, 1, \ldots, 1\right)
$$


Case 2: There exists $k \in\{2, \ldots, p-2\}$ such that

$$
\frac{e_{2}}{e_{1}}=\ldots=\frac{e_{k}}{e_{k-1}} \quad \text { but } \quad \frac{e_{k}}{e_{k-1}} \neq \frac{e_{k+1}}{e_{k}} .
$$

Then, as in Case 2 of the proof of Proposition 3.3, we define

$$
g_{k}=\left(b^{a^{p-k+1}}\right)^{e_{k}}\left(b^{a^{p-k}}\right)^{-e_{k-1}} \in \operatorname{Stab}_{G}(1)
$$

so that

$$
\psi_{1}\left(g_{k}\right)=\left(a^{f_{1}}, \ldots, a^{f_{p-k-1}}, a^{f_{p-k}} b^{-e_{k-1}}, b^{e_{k}} a^{f_{p-k+1}}, 1, \frac{k-1}{.}, 1\right),
$$

where

$$
\begin{aligned}
f_{1} & =e_{k}^{2}-e_{k-1} e_{k+1} \neq 0, \\
f_{l} & =e_{k} e_{k+l-1}-e_{k-1} e_{k+l} \quad \text { for } 2 \leq l \leq p-k-1, \\
f_{p-k} & =e_{k} e_{p-1} \neq 0 \quad \text { and } \quad f_{p-k+1}=-e_{k-1} \neq 0 .
\end{aligned}
$$

The first statement follows from $(3.2)$, while the last two are due to the circumstance that $e_{1}, \ldots, e_{p-1} \neq 0$.

We now identify a suitable conjugate $g$ of $g_{k}$. First we take any conjugate $h$ of $g_{k}$ by a power of $a$ such that the $j$ th entry of $\psi_{1}(h)$ does not involve a non-trivial power of $a$. Hence the $j$ th entry is trivial by the form of $\psi_{1}\left(g_{k}\right)$. If the $i$ th entry of $\psi_{1}(h)$ also does not involve a non-trivial power of $a$, then consider $h^{a^{l}}$, where $l=j-i$. Now the $j$ th entry of $\psi_{1}\left(h^{a^{l}}\right)$ is trivial. If the $i$ th entry of $\psi_{1}\left(h^{a^{l}}\right)$ does not involve a non-trivial power of $a$, then repeat the process and consider $h^{a^{2 l}}$, etc. Since there are entries of $h$ that involve non-trivial powers of $a$, we arrive in this way at $g=h^{a^{m l}}$, for some $m \in\{0,1, \ldots, p-1\}$, such that the $j$ th entry of $\psi_{1}(g)$ is trivial, while the $i$ th entry involves a non-trivial power of $a$. Thus condition $(\dagger)$ is again satisfied.

We remark that GGS-groups $G=\langle a, b\rangle$ are branch apart from when the defining vector of the directed automorphism $b$ is constant. In the latter case the group $G$ is only weakly branch, that is, all rigid stabilisers are non-trivial; see [9, Theorem 3.7] and [10, Lemma 4.2(iii)]. The next corollary extends the results in 14 which cover GGS-groups with defining vector having at least one zero entry, and additionally the Gupta-Sidki 3-group.

Corollary 3.8. Let $G \in \mathscr{C}$ be branch. Then the branch action of $G$ is unique in the sense of Theorem 3.6.

Proof. Observe that $G$ contains a branch GGS-group. Thus Proposition 3.7 and [14, Lemmata 6 and 7] imply that $G$, too, satisfies condition $(\dagger)$. Hence the result follows by Theorem 3.6 . 
3.2. Length functions and abelianisation. For the proof of part (2) of Theorem 1.4 we require certain length functions on the groups $G \in \mathscr{C}$; as a by-product we pin down the abelianisation $G / G^{\prime}$ of $G$. Fix a group $G=\left\langle a, \mathbf{b}^{(1)}, \ldots, \mathbf{b}^{(p)}\right\rangle \in \mathscr{C}$ in standard form and consider the free product

$$
\widehat{G}=\langle\hat{a}\rangle *\left\langle\widehat{\mathbf{b}}^{(1)}\right\rangle * \ldots *\left\langle\widehat{\mathbf{b}}^{(p)}\right\rangle
$$

of elementary abelian $p$-groups $\langle\hat{a}\rangle \cong C_{p}$ and $\left\langle\widehat{\mathbf{b}}^{(j)}\right\rangle=\left\langle\hat{b}_{1}^{(j)}, \ldots, b_{r_{j}}^{(j)}\right\rangle \cong C_{p}^{r_{j}}$ for $1 \leq j \leq p$. Note that there is a unique epimorphism $\pi: \widehat{G} \rightarrow G$ such that $\hat{a} \mapsto a$ and $\hat{b}_{i}^{(j)} \mapsto b_{i}^{(j)}$ for $1 \leq j \leq p$ and $1 \leq i \leq r_{j}$, inducing an epimorphism from $\widehat{G} / \widehat{G}^{\prime} \cong C_{p}^{1+r_{1}+\ldots+r_{p}}$ onto $G / G^{\prime}$. We want to show that the latter is an isomorphism; see Proposition 3.9.

By the standard theory of free products of groups, each element $\hat{g} \in \widehat{G}$ has a unique reduced form

$$
\hat{g}=\hat{a}^{\alpha_{1}} w_{1} \hat{a}^{\alpha_{2}} w_{2} \cdots \hat{a}^{\alpha_{l}} w_{l} \hat{a}^{\alpha_{l+1}},
$$

where $l \in \mathbb{N} \cup\{0\}, w_{1}, \ldots, w_{l} \in\left\langle\widehat{\mathbf{b}}^{(1)} \cup \ldots \cup \widehat{\mathbf{b}}^{(p)}\right\rangle \backslash\{1\}$, and $\alpha_{1}, \ldots, \alpha_{l+1} \in$ $\mathbb{Z} / p \mathbb{Z}$ such that $\alpha_{i} \neq 0$ for $i \in\{2, \ldots, l\}$. Furthermore, for each $i \in$ $\{1, \ldots, l\}$, the element $w_{i}$ can be uniquely expressed as

$$
w_{i}=\left(\widehat{\mathbf{b}}^{(k(i, 1))}\right)^{\boldsymbol{\beta}(i, 1)} \cdots\left(\widehat{\mathbf{b}}^{\left(k\left(i, n_{i}\right)\right)}\right)^{\boldsymbol{\beta}\left(i, n_{i}\right)},
$$

where $n_{i} \in \mathbb{N}, k(i, 1), \ldots, k\left(i, n_{i}\right) \in\{1, \ldots, p\}$, with $k(i, m) \neq k(i, m+1)$ for $1 \leq m \leq n_{i}-1$, and

$$
\boldsymbol{\beta}(i, m)=\left(\beta(i, m)_{1}, \ldots, \beta(i, m)_{r_{k(i, m)}}\right) \in(\mathbb{Z} / p \mathbb{Z})^{r_{k(i, m)}} \backslash\{\mathbf{0}\}, \quad 1 \leq m \leq n_{i},
$$

are exponent vectors so that

$$
\left(\widehat{\mathbf{b}}^{(k(i, m))}\right)^{\boldsymbol{\beta}(i, m)}=\left(\hat{b}_{1}^{(k(i, m))}\right)^{\beta(i, m)_{1}} \cdots\left(\hat{b}_{r_{k(i, m)}}^{(k(i, m))}\right)^{\beta(i, m)_{r_{k(i, m)}} .}
$$

The length of $\hat{g}$ is defined as

$$
\partial(\hat{g})=n_{1}+\ldots+n_{l} .
$$

Furthermore, we define exponent maps from $\widehat{G}$ to $\mathbb{Z} / p \mathbb{Z}$ by

$$
\begin{aligned}
\varepsilon_{\hat{a}}(\hat{g}) & =\sum_{m=1}^{l+1} \alpha_{m} \text { and } \\
\varepsilon_{\hat{b}_{j}^{(k)}}(\hat{g}) & =\sum_{\substack{1 \leq i \leq l, 1 \leq m \leq n_{i} \\
\text { s.t. } k(i, m)=k}} \beta(i, m)_{j} \quad \text { for } 1 \leq k \leq p \text { and } 1 \leq j \leq r_{k} .
\end{aligned}
$$

The isomorphism $G /[G, G] \cong \widehat{G} /[\widehat{G}, \widehat{G}]$ is obtained parallel to [1, Section 4.1], which uses a reformulation of Rozhkov [24].

Proposition 3.9. Let $G \in \mathscr{C}$ be in standard form and $\widehat{G}$ as above. Then the surjective homomorphism

$$
\widehat{G} \rightarrow(\mathbb{Z} / p \mathbb{Z}) \times \prod_{j=1}^{p}(\mathbb{Z} / p \mathbb{Z})^{r_{j}}, \quad \hat{g} \mapsto\left(\varepsilon_{\hat{a}}(\hat{g}),\left(\left(\varepsilon_{\hat{b}_{i}^{(1)}}(\hat{g})\right)_{i=1}^{r_{1}}, \ldots,\left(\varepsilon_{\hat{b}_{i}^{(p)}}(\hat{g})\right)_{i=1}^{r_{p}}\right)\right)
$$


with kernel $\widehat{G}^{\prime}$ factors through $G / G^{\prime}$ and consequently,

$$
\begin{aligned}
G / G^{\prime} & \cong\left\langle\underline{a}, \underline{b}_{1}^{(1)}, \ldots, \underline{b}_{r_{1}}^{(1)}, \quad \ldots \quad \ldots, \quad \underline{b}_{1}^{(p)}, \ldots, \underline{b}_{r_{p}}^{(p)}\right\rangle \\
& \cong C_{p} \times{ }^{1+r_{1}+\ldots+r_{p}} \times C_{p} .
\end{aligned}
$$

Let $G \in \mathscr{C}$ and $\pi: \widehat{G} \rightarrow G$ be the natural epimorphism as above. The length of $g \in G$ is

$$
\partial(g)=\min \left\{\partial(\hat{g}) \mid \hat{g} \in \pi^{-1}(g)\right\} .
$$

Moreover, via Proposition 3.9, we define

$$
\varepsilon_{a}(g)=\varepsilon_{\hat{a}}(\hat{g}) \text { and } \varepsilon_{b_{i}^{(j)}}(g)=\varepsilon_{\hat{b}_{i}^{(j)}}(\hat{g}) \text {, for } 1 \leq j \leq p \text { and } 1 \leq i \leq r_{j},
$$

via any pre-image $\hat{g} \in \pi^{-1}(g)$.

Since $G$ is fractal, every $g \in G$ may be expressed as

$$
g=\psi_{1}^{-1}\left(g_{1}, \ldots, g_{p}\right) a^{\varepsilon_{a}(g)},
$$

where $g_{i} \in G$ for $1 \leq i \leq p$. Of course, the decomposition can be applied repeatedly, yielding, for instance, $g_{i}=\psi_{1}^{-1}\left(g_{i, 1}, \ldots, g_{i, p}\right) a^{\varepsilon_{a}\left(g_{i}\right)}$ for $1 \leq i \leq p$.

Lemma 3.10. Let $G=\left\langle a, \mathbf{b}^{(1)}, \ldots, \mathbf{b}^{(p)}\right\rangle \in \mathscr{C}$ be in standard form, and let $g \in G$. Then, using the notation introduced above, $\sum_{i=1}^{p} \partial\left(g_{i}\right) \leq \partial(g)$.

Suppose further that $\partial(g)>1$. Then $\partial\left(g_{i, j}\right)<\partial(g)$ for all $i, j \in\{1, \ldots, p\}$.

Proof. Let $\partial(g)=m$. We may express $g a^{-\varepsilon_{a}(g)}=\psi_{1}^{-1}\left(g_{1}, \ldots, g_{p}\right)$ as

$$
\left(\left(\mathbf{b}^{(k(1))}\right)^{\boldsymbol{\beta}(1)}\right)^{a^{e_{1}}}\left(\left(\mathbf{b}^{(k(2)}\right)^{\boldsymbol{\beta}(2)}\right)^{a^{e_{2}}} \cdots\left(\left(\mathbf{b}^{(k(m))}\right)^{\boldsymbol{\beta}(m)}\right)^{a^{e_{m}}}
$$

with $k(i) \in\{1, \ldots, p\}, e_{i} \in \mathbb{Z} / p \mathbb{Z}$ and exponent vectors $\boldsymbol{\beta}(i) \in(\mathbb{Z} / p \mathbb{Z})^{r_{k(i)}} \backslash$ $\{\mathbf{0}\}$ for $1 \leq i \leq m$. Furthermore, we have $k(i) \neq k(i+1)$ whenever $e_{i}=e_{i+1}$. For each $i \in\{1, \ldots, m\}$, the factor $\left(\left(\mathbf{b}^{(k(i))}\right)^{\boldsymbol{\beta}(i)}\right)^{a^{e_{i}}}$ contributes to precisely one coordinate $g_{j(i)}$ a factor $\left(\mathbf{b}^{(k(i))}\right)^{\boldsymbol{\beta}(i)}$ and to all other coordinates $g_{l}$, $l \neq j(i)$, a power of $a$. Hence the first statement of the lemma follows.

Suppose that $\partial\left(g_{i, j}\right)=\partial(g)$ for some $i, j \in\{1, \ldots, p\}$. This implies $\partial\left(g_{i}\right)=\partial(g)$ and

$$
g_{i}=\left(\mathbf{b}^{(k(1))}\right)^{\boldsymbol{\beta}(1)}\left(\mathbf{b}^{(k(2))}\right)^{\boldsymbol{\beta}(2)} \cdots\left(\mathbf{b}^{(k(m))}\right)^{\boldsymbol{\beta}(m)} .
$$

Now either $k(1)=\ldots=k(m)$ and then $\partial(g)=\partial\left(g_{i}\right) \in\{0,1\}$, or there exists $l \in\{1, \ldots, m-1\}$ such that $k(l) \neq k(l+1)$ and thus $\partial\left(g_{i}\right)>\partial\left(g_{i, j}\right)$ for $1 \leq j \leq p$.

We now prove part (3) of Theorem 1.4

Proposition 3.11. Let $G=\left\langle a, \mathbf{b}^{(1)}, \ldots, \mathbf{b}^{(p)}\right\rangle \in \mathscr{C}$ be in standard form and such that the families $\mathbf{b}^{(1)}, \ldots, \mathbf{b}^{(p)}$ of directed generators of $G$ satisfy the additional conditions

(i) every non-empty family $\mathbf{b}^{(j)}, j \in\{1, \ldots, p\}$, features at least one non-symmetric defining vector; 
(ii) there are at least two directed automorphisms, from two distinct families, that have the same defining vector.

Then $G$ does not have the congruence subgroup property.

Proof. Based on condition (ii), we find directed generators $b \in \mathbf{b}^{(i)}$ and $c \in \mathbf{b}^{(j)}$, where $1 \leq i<j \leq p$, and $\left(e_{1}, \ldots, e_{p-1}\right) \in(\mathbb{Z} / p \mathbb{Z})^{p-1}$ such that $b=\left(a^{e_{i}}, \ldots, a^{e_{p-1}}, b, a^{e_{1}}, \ldots, a^{e_{i-1}}\right), \quad c=\left(a^{e_{j}}, \ldots, a^{e_{p-1}}, c, a^{e_{1}}, \ldots, a^{e_{j-1}}\right)$.

We proceed as in [21, Lemma 3.1 and Corollary 3.1].

First we construct recursively, for $n \in \mathbb{N}$, elements $t_{n} \in b G^{\prime} \cap c \operatorname{Stab}_{G}(n)$. Set $t_{1}=b$. For $n \geq 2$, suppose $t_{n-1} \in b G^{\prime} \cap c \operatorname{Stab}_{G}(n-1)$. Condition (i) tells us that Proposition 3.4 is at our disposal so that

$$
x_{n}:=\psi_{1}^{-1}\left(1, \stackrel{p-j}{-}, 1, b^{-1} t_{n-1}, 1, \stackrel{j-1}{\cdots}, 1\right) \in G^{\prime} .
$$

Setting $t_{n}=b^{a^{i-j}} x_{n} \in b G^{\prime}$, we conclude that

$$
\psi_{1}\left(t_{n}\right)=\left(a^{e_{j}}, \ldots, a^{e_{p-1}}, t_{n-1}, a^{e_{1}}, \ldots, a^{e_{j-1}}\right)
$$

and thus

$$
\psi_{1}\left(c^{-1} t_{n}\right)=\left(1, \stackrel{p-j}{\cdot}, 1, c^{-1} t_{n-1}, 1, \stackrel{j-1}{-1}, 1\right) .
$$

Since $c^{-1} t_{n-1} \in \operatorname{Stab}_{G}(n-1)$, we see that $c^{-1} t_{n} \in \operatorname{Stab}_{G}(n)$, and hence $t_{n} \in b G^{\prime} \cap c \operatorname{Stab}_{G}(n)$.

To finish, we prove that the finite-index subgroup $G^{\prime}$ of $G$ is not a congruence subgroup, i.e. does not contain $\operatorname{Stab}_{G}(n)$ for any $n \in \mathbb{N}$. From Proposition 3.9 , it follows that $c^{-1} t_{n} \equiv c^{-1} b \not \equiv 1\left(\bmod G^{\prime}\right)$. Hence $G^{\prime}$ does not contain $\operatorname{Stab}_{G}(n)$.

3.3. Weakly maximal subgroups. Let $G \in \mathscr{C}$ be branch. We recall that the parabolic subgroups of $G$ are the stabilisers of the boundary points $\ell \in \partial T$. A subgroup of $G$ is weakly maximal if it is maximal among the subgroups of infinite index. For a finitely generated regular branch group, [6. Theorem 1.1] shows that any finite subgroup is contained in uncountably many weakly maximal subgroups. It then follows that there are uncountably many non-parabolic weakly maximal subgroups. This applies accordingly to $G$, by using the finite subgroup $\langle a\rangle$ acting fix-point freely on $\partial T$. Note also that Corollary 3.8 allows us to consider all branch actions, thus [6, Theorem 1.3] is partly generalised:

Corollary 3.12. Let $G \in \mathscr{C}_{\text {reg. }}$. Then there exist uncountably many $\operatorname{Aut}(G)$ orbits of weakly maximal subgroups of $G$, all distinct from the orbits of parabolic subgroups under any branch action of $G$ on any spherically homogeneous rooted tree.

\section{THETA MAPS}

4.1. Length reduction. Let $G=\left\langle a, \mathbf{b}^{(1)}, \ldots, \mathbf{b}^{(p)}\right\rangle \in \mathscr{C}$ be in standard form. We may assume that $r_{1} \neq 0$ and that $b_{1}=b_{1}^{(1)}$ satisfies

$$
\psi_{1}\left(b_{1}\right)=\left(a^{e_{1,1}}, \ldots, a^{e_{1, p-1}}, b_{1}\right)=\left(a, a^{e_{1,2}}, \ldots, a^{e_{1, p-1}}, b_{1}\right) ;
$$


see Lemma 3.1, We set

$$
n=\max \left\{j \in\{1, \ldots, p-1\} \mid e_{1, j} \neq 0 \text { in } \mathbb{Z} / p \mathbb{Z}\right\} .
$$

Whereas we considered a slightly more general setting in [1], we suppose here from the outset that $G$ is a torsion group so that $n \geq 2$. This shortens some of the proofs.

In preparation for Section 5, we recall from [1, Section 4.2] two length decreasing maps $\Theta_{1}, \Theta_{2}: G^{\prime} \rightarrow G^{\prime}$. Clearly, $G^{\prime}$ is a subgroup of $\operatorname{Stab}_{G}(1)$. Furthermore, every $g \in \operatorname{Stab}_{G}(1)$ has a decomposition

$$
\psi_{1}(g)=\left(g_{1}, \ldots, g_{p}\right),
$$

where each $g_{j} \in U_{u_{j}}^{G} \cong G$ is an element of the upper companion group acting on the subtree rooted at a first level vertex $u_{j}, j \in\{1, \ldots, p\}$, and we define

$$
\varphi_{j}: \operatorname{Stab}_{G}(1) \rightarrow \operatorname{Aut}\left(T_{u_{j}}\right) \cong \operatorname{Aut}(T), \quad \varphi_{j}(g)=g_{j},
$$

using the natural identification of $T_{u_{j}}$ and $T$. In the proof of Theorem 4.1 below we write $\left(g_{1}, \ldots, g_{p}\right)$ without warning in place of $g \in \operatorname{Stab}_{G}(1)$, as is customary to streamline certain computations.

We are interested in projecting, via $\varphi_{p}$, the first level stabiliser $\operatorname{Stab}_{M}(1)$ of a subgroup $M \leq G$, containing $b_{1}$ and an 'approximation' $a z \in a G^{\prime}$ of $a$, to a subgroup of $\operatorname{Aut}\left(T_{u_{p}}\right)$. Writing $\psi_{1}(z)=\left(z_{1}, \ldots, z_{p}\right)$, one can show that

$$
\varphi_{p}\left(b_{1}{ }^{(a z)^{-1}}\right)=a^{z_{1}^{-1}}=a\left[a, z_{1}^{-1}\right]
$$

and from this we define

$$
\Theta_{1}: G^{\prime} \rightarrow G^{\prime}, \quad \Theta_{1}(z)=\left[a, z_{1}^{-1}\right] .
$$

The map $\Theta_{2}$ is obtained similarly. As $e_{1, n} \neq 0$, we find $k \in \mathbb{Z} / p \mathbb{Z}$ such that $k e_{1, n}=1$. One can show that

$$
\varphi_{p}\left(\left(b_{1}{ }^{k}\right)^{(a z)^{p-n}}\right)=a^{z_{n+1} \cdots z_{p}}=a\left[a, z_{n+1} \cdots z_{p}\right]
$$

and we define

$$
\Theta_{2}: G^{\prime} \rightarrow G^{\prime}, \quad \Theta_{2}(z)=\left[a, z_{n+1} \ldots z_{p}\right] .
$$

Theorem 4.1. Let $G=\left\langle a, \mathbf{b}^{(1)}, \ldots, \mathbf{b}^{(p)}\right\rangle \in \mathscr{C}$ be in standard form such that $r_{1} \neq 0$ and (4.1) holds. Suppose further that $G$ is a torsion group. Then the length $\partial(z)$ of an element $z \in G^{\prime}$ decreases under repeated applications of a suitable combination of the maps $\Theta_{1}$ and $\Theta_{2}$ down to length 0 or 2.

Proof. Let $z \in G^{\prime}$. We observe that $\partial(z) \neq 1$. Suppose that $m=\partial(z) \geq 3$. Then $z \in G^{\prime} \subseteq \operatorname{Stab}_{G}(1)$ has a decomposition

$$
\psi_{1}(z)=\left(z_{1}, \ldots, z_{p}\right)
$$

and Lemma 3.10 yields

$$
\partial\left(z_{1}\right)+\partial\left(z_{n+1} \cdots z_{p}\right) \leq m,
$$

where $n \geq 2$ is as defined in 4.2. 
If $\partial\left(z_{1}\right)<\frac{m}{2}$ then $\partial\left(\Theta_{1}(z)\right)<m$, and likewise if $\partial\left(z_{n+1} \cdots z_{p}\right)<\frac{m}{2}$ then $\partial\left(\Theta_{2}(z)\right)<m$, and we apply induction. Hence we may suppose that $m=2 \mu$ is even and

$$
\partial\left(z_{1}\right)=\partial\left(z_{n+1} \cdots z_{p}\right)=\mu .
$$

We write $Z=z_{n+1} \cdots z_{p}=a^{k}\left(Z_{1}, \ldots, Z_{p}\right)$, for suitable $k \in \mathbb{Z} / p \mathbb{Z}$ and $\left(Z_{1}, \ldots, Z_{p}\right) \in \operatorname{Stab}_{G}(1)$ with $\sum_{i=1}^{p} \partial\left(Z_{i}\right) \leq \mu$; see Lemma 3.10. Consider

$$
\Theta_{2}(z)=[a, Z]=Z^{-a} Z=\left(Z_{p}^{-1} Z_{1}, Z_{1}^{-1} Z_{2}, \ldots, Z_{p-1}^{-1} Z_{p}\right) .
$$

If $\partial\left(Z_{1}\right) \geq 1$, then $n \geq 2$ implies

$$
\partial\left(\left(\Theta_{2}(z)\right)_{1}\right)+\partial\left(\left(\Theta_{2}(z)\right)_{n+1} \cdots\left(\Theta_{2}(z)\right)_{p}\right)=\partial\left(Z_{p}^{-1} Z_{1}\right)+\partial\left(Z_{n}^{-1} Z_{p}\right)<m .
$$

Consequently, $\partial\left(\left(\Theta_{2}(z)\right)_{1}\right)<\frac{m}{2}$ or $\partial\left(\left(\Theta_{2}(z)\right)_{n+1} \cdots\left(\Theta_{2}(z)\right)_{p}\right)<\frac{m}{2}$ and we are done by our earlier argument.

From now on suppose that $Z_{1} \in\langle a\rangle$. Applying (4.4) to $\Theta_{2}(z)$ instead of $z$, we may assume that $\partial\left(Z_{p}\right)=\partial\left(\left(\Theta_{2}(z)\right)_{1}\right)=\mu$. This implies that, in our usual notation,

$$
Z=a^{k}\left(\left(\mathbf{b}^{(k(1))}\right)^{\boldsymbol{\beta}(1)}\right)^{a^{*}}\left(\left(\mathbf{b}^{(k(2))}\right)^{\boldsymbol{\beta}(2)}\right)^{a^{*}} \cdots\left(\left(\mathbf{b}^{(k(\mu))}\right)^{\boldsymbol{\beta}(\mu)}\right)^{a^{*}},
$$

where $k(i) \in\{1, \ldots, p\}$ for $1 \leq i \leq \mu$, the $\boldsymbol{\beta}(i) \in(\mathbb{Z} / p \mathbb{Z})^{r_{k(i)}}$ are suitable exponent vectors and the undeclared exponents $*$ of $a$ are such that

$$
Z_{p}=\left(\mathbf{b}^{(k(1))}\right)^{\boldsymbol{\beta}(1)}\left(\mathbf{b}^{(k(2))}\right)^{\boldsymbol{\beta}(2)} \cdots\left(\mathbf{b}^{(k(\mu))}\right)^{\boldsymbol{\beta}(\mu)}
$$

and $\left\{Z_{1}, \ldots, Z_{p-1}\right\} \subseteq\langle a\rangle$; furthermore, $k(i) \neq k(i+1)$ for $1 \leq i \leq \mu-1$. This implies $Z_{n}^{-1} Z_{p}=a^{l} Z_{p}$ for some $l \in \mathbb{Z} / p \mathbb{Z}$, hence

$$
\Theta_{2}\left(\Theta_{2}(z)\right)=\left[a, a^{l} Z_{p}\right]=\left[a, Z_{p}\right]=Z_{p}^{-a} Z_{p}
$$

We now repeat, for $Z_{p}=\left(Z_{p, 1}, \ldots, Z_{p, p}\right)$, the argument applied earlier to $Z$. If $\partial\left(Z_{p, 1}\right) \geq 1$, we are done by our earlier reasoning. Otherwise we see that $\partial\left(Z_{p, p}\right)=\mu$, and 4.5 implies $k(1)=k(2)=\ldots=k(\mu)=1$ leading to $\mu=1$, hence $m=2 \mu=2$, a contradiction.

We briefly comment that the above proof simplifies the corresponding proof in [1, Theorem 4.5] for the multi-edge spinal groups when $n \geq 2$.

\section{Maximal Subgroups}

The cosets of finite-index subgroups of a group $G$ form a base for the profinite topology on $G$. A subgroup $H$ of $G$ is dense with respect to the profinite topology if and only if $G=N H$ for every finite-index normal subgroup $N$ of $G$. Thus every maximal subgroup of infinite index in $G$ is dense and every proper dense subgroup is contained in a maximal subgroup of infinite index.

We consider a group $G \leq \operatorname{Aut}(T)$ acting on the regular $p$-adic rooted tree $T$. For any vertex $u$ of $T$, the group $U_{u}^{G} \leq \operatorname{Aut}\left(T_{u}\right)$ maps isomorphically onto a group $G_{u} \leq \operatorname{Aut}(T)$ under the map induced by the natural identification of $T_{u}$ with $T$. Similarly, for a subgroup $M$ of $G$, we write 
$M_{u}$ for the corresponding image of $U_{u}^{M}$. If $G$ is fractal, then $G_{u}=G$ but nevertheless it is sometimes useful to write $G_{u}$ to emphasise that we are considering the isomorphic image of $U_{u}^{G}$.

Taking into consideration that the notational convention in [1] is slightly different, we record some preliminary results.

Proposition 5.1 ([20, Proposition 3.2]). Let $T$ be a spherically homogeneous rooted tree and let $G \leq \operatorname{Aut}(\mathrm{T})$ be a just infinite group acting transitively on each level of $T$. Let $M$ be a dense subgroup of $G$ with respect to the profinite topology. Then

(1) the subgroup $M$ acts transitively on each level of the tree $T$,

(2) for every vertex $u \in T$, the subgroup $M_{u}$ is dense in $G_{u}$ with respect to the profinite topology.

The following result is a direct generalisation of [1, Proposition 5.2]. The proof of the latter, however, does not seem to contain all necessary details; these have now been worked out in a more general setting by Francoeur and Garrido following a strategy originally due to Pervova; see [11, Proposition 6.3].

Proposition 5.2. Let $T$ be the regular $p$-adic rooted tree, and let $G \leq$ Aut $(T)$ be a branch group that is just infinite and fractal. Let $M$ be a proper dense subgroup of $G$, with respect to the profinite topology. Then $M_{u}$ is a proper subgroup of $G_{u}=G$ for every vertex $u$ of $T$.

We now proceed in similar fashion to [1].

Proposition 5.3. Let $G=\left\langle a, \mathbf{b}^{(1)}, \ldots, \mathbf{b}^{(p)}\right\rangle \in \mathscr{C}$ be in standard form. Suppose that $G$ is a torsion group, and let $M$ be a dense subgroup of $G$, with respect to the profinite topology.

Then for each $j \in\{1, \ldots, p\}$ and $i \in\left\{1, \ldots, r_{j}\right\}$ there is a vertex $u$ of $T$ and an element $g \in \operatorname{Stab}_{G}(u)$, acting on $T_{u}$ as $a^{\ell}$ for some $\ell \in \mathbb{Z} / p \mathbb{Z}$ under the natural identification of $T_{u}$ and $T$, such that

(i) $\left(M^{g}\right)_{u}=\left(M_{u}\right)^{a^{\ell}}$ is a dense subgroup of $G_{u}=G$,

(ii) there exists $c \in\left(M_{u}\right)^{a^{\ell}} \cap\left\langle b_{1}^{(j)}, \ldots, b_{r_{j}}^{(j)}\right\rangle$ such that $\varepsilon_{b_{i}^{(j)}}(c) \neq 0$.

Proof. By symmetry it suffices to prove the statement for $j=1$ and $i=1$, assuming $r_{1}>0$. For notational simplicity, we write $b_{1}=b_{1}^{(1)}, \ldots, b_{r_{1}}=b_{r_{1}}^{(1)}$. It suffices to produce $u$ such that (ii) holds, as with $G$ being fractal, the existence of $g$ is automatic and Proposition 5.1 yields (i).

Since $\left|G: G^{\prime}\right|$ is finite, $G^{\prime}$ is open in the profinite topology. Thus we find $x \in M \cap b_{1} G^{\prime}$. In particular $x \in \operatorname{Stab}_{G}(1)$ with $\varepsilon_{b_{1}}(x) \neq 0$ in $\mathbb{Z} / p \mathbb{Z}$. We argue by induction on $\partial(x) \geq 1$.

First suppose that $\partial(x)=1$. Then $x$ has the form $x=c^{a^{\ell}}$, where $c \in$ $\left\langle b_{1}, \ldots, b_{r_{1}}\right\rangle$ with $\varepsilon_{b_{1}}(c) \neq 0$. Thus choosing the vertex $u$ to be the root of the tree $T$, we have $c \in M^{a^{-\ell}}=\left(M_{u}\right)^{a^{-\ell}}$. 
Now suppose that $m=\partial(x) \geq 2$. We first determine a suitable vertex $u_{\omega}$ at level 1 or 2 , as follows. Observe that

$$
\varepsilon_{b_{1}}\left(\varphi_{1}(x)\right)+\ldots+\varepsilon_{b_{1}}\left(\varphi_{p}(x)\right)=\varepsilon_{b_{1}}(x) \neq 0 .
$$

If there exists $i \in\{1, \ldots, p\}$ with $\varepsilon_{b_{1}}\left(\varphi_{i}(x)\right) \neq 0$ and $\partial\left(\varphi_{i}(x)\right)<\partial(x)$, then we fix $\omega=i$. If not, then for each $j \in\{1, \ldots, p\}$ with $\varepsilon_{b_{1}}\left(\varphi_{j}(x)\right) \neq 0$ we have $\partial\left(\varphi_{j}(x)\right)=\partial(x)$. But Lemma 3.10 shows that $\sum_{i=1}^{p} \partial\left(\varphi_{i}(x)\right) \leq \partial(x)$; hence there exists a unique $j$ such that $\partial\left(\varphi_{j}(x)\right)=\partial(x)$ and $\varphi_{i}(x) \in\langle a\rangle$ for all remaining indices $i \neq j$. This implies that $x$ is of the form

$$
x=\left(\left(\mathbf{b}^{(k(1))}\right)^{\boldsymbol{\beta}(1)}\right)^{a^{*}} \cdots\left(\left(\mathbf{b}^{(k(m))}\right)^{\boldsymbol{\beta}(m)}\right)^{a^{*}},
$$

where the exponents $*$ are such that $\varphi_{j}(x)=\left(\mathbf{b}^{(k(1))}\right)^{\boldsymbol{\beta}(1)} \cdots\left(\mathbf{b}^{(k(m))}\right)^{\boldsymbol{\beta}(m)} \in$ $\operatorname{Stab}_{G}(1)$. Furthermore $\varepsilon_{b_{1}}\left(\varphi_{j}(x)\right) \neq 0$ implies that $\varepsilon_{b_{1}}\left(\varphi_{j p}(x)\right) \neq 0$ and $\partial\left(\varphi_{j p}(x)\right)<\partial(x)$, where $\varphi_{j p}(x)=\varphi_{p}\left(\varphi_{j}(x)\right)$. We fix $\omega=j p$.

We proceed in our analysis with

$$
\tilde{x}=\varphi_{\omega}(x) \in M_{u_{\omega}} \leq G_{u_{\omega}}=G,
$$

satisfying $\varepsilon_{b_{1}}(\tilde{x}) \neq 0$ and $\partial(\tilde{x})<\partial(x)$. First suppose that $\tilde{x} \in \operatorname{Stab}_{G_{u(\omega)}}(1)$, where we write $u(\omega)=u_{\omega}$ for readability. By Proposition 5.2, the subgroup $M_{u_{\omega}}$ is dense in $G_{u_{\omega}}=G$, and the result follows by induction.

Now suppose that $\tilde{x} \notin \operatorname{Stab}_{G_{u(\omega)}}(1)$. For $l \in\{1, \ldots, p\}$ we claim

$$
\varepsilon_{b_{1}}\left(\varphi_{l}\left(\tilde{x}^{p}\right)\right)=\varepsilon_{b_{1}}(\tilde{x}) \neq 0 .
$$

To see this, observe that $\tilde{x}$ is of the form

$$
\tilde{x}=a^{k} h=a^{k}\left(h_{1}, \ldots, h_{p}\right),
$$

where $k=\varepsilon_{a}(x) \neq 0$ and $h \in \operatorname{Stab}_{G_{u(\omega)}}(1)$ with $\psi_{1}(h)=\left(h_{1}, \ldots, h_{p}\right)$. Raising $\tilde{x}$ to the prime power $p$, we obtain

$$
\tilde{x}^{p}=\left(a^{k} h\right)^{p}=h^{a^{(p-1) k}} \cdots h^{a^{k}} h,
$$

and thus, for $l \in\{1, \ldots, p\}$,

$$
\varphi_{l}\left(\tilde{x}^{p}\right) \equiv h_{1} h_{2} \cdots h_{p} \quad\left(\bmod G_{u_{\omega}}^{\prime}\right) .
$$

In view of (5.2) and (5.3), we conclude that (5.1) holds.

Furthermore we observe, from the above equations and from the proof of Lemma 3.10, that

$$
\partial\left(\varphi_{l}\left(\tilde{x}^{p}\right)\right) \leq \partial(\tilde{x})<\partial(x)
$$

If $\varphi_{l}\left(\tilde{x}^{p}\right) \in M_{u(\omega l)}$ belongs to $\operatorname{Stab}_{G_{u(\omega l)}}(1)$, we are done as before by induction. If not, we apply the operation $y \mapsto \varphi_{l}\left(y^{p}\right)$ repeatedly. Since $M$ is a torsion group, $x \in \operatorname{Stab}_{M}(1)$ and $\tilde{x}$ have finite order. Clearly, if $y \in G$ has finite order then $\varphi_{l}\left(y^{p}\right)$ has order strictly smaller than $y$. Thus after finitely many iterations, we reach an element

$$
\tilde{\tilde{x}}=\varphi_{l}\left(\varphi_{l}\left(\ldots \varphi_{l}\left(\varphi_{\omega}(x)^{p}\right)^{p} \ldots\right)^{p}\right) \in M_{u(\omega l \ldots l)}
$$


which, in addition to the inherited properties $\varepsilon_{b_{1}}(\tilde{\tilde{x}}) \neq 0$ and $\partial(\tilde{\tilde{x}})<\partial(x)$, satisfies $\tilde{\tilde{x}} \in \operatorname{Stab}_{G_{u(\omega l \ldots l)}}(1)$. As before, the proof concludes by induction.

The next result follows as in [1, Proposition 5.4], however we give a slightly conciser version of the proof here.

Proposition 5.4. Let $G=\left\langle a, \mathbf{b}^{(1)}, \ldots, \mathbf{b}^{(p)}\right\rangle \in \mathscr{C}$ be in standard form such that $r_{1} \neq 0$ and (4.1) holds. Suppose further that $G$ is torsion. Let $M$ be a dense subgroup of $G$, with respect to the profinite topology, and suppose that $b_{1}^{(1)} \in M$. Then there exists a vertex $u$ of $T$ and an element $g \in \operatorname{Stab}_{G}(u)$ acting on $T_{u}$ as $h \in \operatorname{Stab}_{G}(1)$ under the natural identification of $T_{u}$ and $T$, such that

(i) $\left(M^{g}\right)_{u}=\left(M_{u}\right)^{h}$ is a dense subgroup of $G_{u}=G$,

(ii) $a, b_{1}^{(1)} \in\left(M_{u}\right)^{h}$.

Proof. It suffices to establish the existence of $u$ and $h$ such that (ii) holds, because $G$ is fractal. Since $G^{\prime}$ is open and $M$ is dense in $G$, there is $z \in G^{\prime}$ such that $a z \in M$. Recall that we denote the $p$ th vertex at level 1 by $u_{p}$. The coordinate map $\varphi_{p}$ allows us to restrict $\operatorname{Stab}_{M}(1)$ to $M_{u_{p}}$, and $b_{1}^{(1)} \in M$ implies $b_{1}^{(1)} \in M_{u_{p}}$.

Consider the theta maps $\Theta_{1}, \Theta_{2}$ defined in Section 4 , and $n \geq 2$ be as in 4.2). By definition, $a \Theta_{1}(z)$ and $a \Theta_{2}(z)$ belong to $M_{u_{p}}$. Moreover, repeated application of $\varphi_{p}$ corresponds to repeated applications of $\Theta_{1}$ and $\Theta_{2}$. By Proposition 5.2 and Theorem 4.1. we may assume that $\partial(z) \in\{0,2\}$.

If $\partial(z)=0$ we take $h=1$ and there is nothing further to prove. Suppose now that $\partial(z)=2$, and write $\psi_{1}(z)=\left(z_{1}, \ldots, z_{p}\right)$. We distinguish between two cases.

Case 1: $\partial\left(z_{1}\right) \in\{0,2\}$. Then $\partial\left(z_{1}\right)=0$ or $\partial\left(z_{n+1} \cdots z_{p}\right)=0$ so that $\Theta_{1}(z)=$ 1 or $\Theta_{2}(z)=1$, and again there is nothing further to prove.

Case 2: $\partial\left(z_{1}\right)=1$. Then $\tilde{z}=\Theta_{1}(z)=z_{1}^{a} z_{1}^{-1}$ satisfies $\partial(\tilde{z})=2$. If $\partial\left(\tilde{z}_{1}\right)=0$ or $\partial\left(\tilde{z}_{n+1} \cdots \tilde{z}_{p}\right)=0$, we proceed as in Case 1 . Thus we may assume that $z_{1}^{-1}=a^{\varepsilon_{a}\left(z_{1}^{-1}\right)}\left(a^{*}, \ldots, a^{*}, h^{-1}\right)$ for suitable $h \in\left\langle\mathbf{b}^{(j)}\right\rangle$ with $j \in\{1, \ldots, p\}$. If $j \neq 1$, then we deduce that $\hat{z}=\Theta_{2}(\tilde{z})=h^{a} h^{-1}$ satisfies $\partial\left(\hat{z}_{1}\right)=0$ or $\partial\left(\hat{z}_{n+1} \cdots \hat{z}_{p}\right)=0$, and we proceed as in Case 1 . Now suppose that $j=1$. Then $h a h^{-1}=a \hat{z}=a \Theta_{2}\left(\Theta_{1}(z)\right)$ and, observing that $b_{1}^{(1)}$ commutes with $h$, we conclude that $a, b_{1}^{(1)} \in\left(M_{u_{p p}}\right)^{h}$.

The proofs of the next two results follow the same logic as those of [1, Proposition 5.5 and Theorem 5.6], so we omit the proofs here.

Proposition 5.5. Let $G \in \mathscr{C}$ be a torsion group, and let $M$ be a dense subgroup of $G$, with respect to the profinite topology. Then there exists a vertex $u$ of $T$ such that $M_{u}=G_{u}=G$. 
Theorem 5.6. Let $G \in \mathscr{C}$ be a torsion group. Then $G$ does not contain any proper dense subgroups, with respect to the profinite topology. Equivalently, $G$ does not contain maximal subgroups of infinite index.

Recall that two groups $G$ and $H$ are (abstractly) commensurable if there exist finite-index subgroups $K \leq G$ and $L \leq H$ with $K \cong L$.

Corollary 5.7. Let $H$ be a group that is commensurable with a torsion group $G \in \mathscr{C}$. Then $H$ does not contain maximal subgroups of infinite index.

Proof. The proof is essentially the same as that of [1, Corollary 1.3]. However we do note here that $G=\left\langle a, \mathbf{b}^{(1)}, \ldots, \mathbf{b}^{(p)}\right\rangle$ contains multi-edge spinal subgroups $G_{j}=\left\langle a, \mathbf{b}^{(j)}\right\rangle$ for $j \in\{1, \ldots, p\}$ with $r_{j} \neq 0$. Within these lie the

associated GGS-groups $G_{j, i}=\left\langle a, b_{i}^{(j)}\right\rangle$ for $i \in\left\{1, \ldots, r_{j}\right\}$. These associated GGS-groups feature in the proof, as in [1].

We have established part (2) of Theorem 1.4 .

\section{IRREDUCIBLE REPRESENTATIONS}

In this section we prove Theorem 1.5 and Theorem 1.7. Throughout, let $F$ denote the prime field $\mathbb{Z} / p \mathbb{Z}$. Let $G \in \mathscr{C}$ be just infinite, acting on the $p$-adic regular tree $T$. We combine the strategies laid out in [2, 25] to demonstrate when the tree enveloping algebra $\mathfrak{A}_{G}$, a proper ring quotient of $F[G]$, is primitive. The latter will imply that $G$ has faithful irreducible representations over $F$.

6.1. Preliminaries. We consider the $F$-vector space $F\langle\langle\partial T\rangle\rangle$ on the basis $\partial T$, the boundary of $T$. The action of $G$ on $\partial T$ extends to an $F$-linear representation of the group algebra

$$
\chi: F[G] \rightarrow \operatorname{End}(F\langle\langle\partial T\rangle\rangle)
$$

which is injective on $G$. The tree enveloping algebra of $G$ is the image $\mathfrak{A}_{G}$ of $F[G]$ under $\chi$. It was implicitly introduced by Sidki [25], albeit in a different form.

We collect some properties of the $F$-algebra $\mathfrak{A}_{G}$ from [2], with statements adapted to our setting. For conciseness we include certain definitions and proofs, though altered to suit our notation and purposes.

Recall that an $F$-algebra $A$ is called just infinite, if $\operatorname{dim}_{F} A=\infty$ and every non-zero two-sided ideal has finite codimension. The Jacobson radical $\operatorname{Jac}(A)$ is the two-sided ideal $\operatorname{Jac}(A)=\bigcap_{X} \operatorname{Ann}(X)$, where $X$ ranges over all simple right $A$-modules. For a one-sided ideal $I$ of $A$, the maximal two-sided ideal contained in $I$ is called the core of $I$, denoted by core $(I)$. The algebra $A$ is primitive if it has a faithful irreducible right module, or equivalently a maximal right ideal with trivial core. The algebra $A$ is semiprimitive if its Jacobson radical is trivial. Finally, let $\operatorname{Aug}\left(\mathfrak{A}_{G}\right)$ denote the image of the augmentation ideal of $F[G]$ in $\mathfrak{A}_{G}$. 
Lemma 6.1 ([2, Lemma 3.8, Theorem 3.9]). Let $G \in \mathscr{C}$ be just infinite. Then its tree enveloping algebra $\mathfrak{A}_{G}$ is just infinite.

Corollary 6.2 ([2, Lemma 3.15] and [25, Corollary 4.4.3]). Let $G \in \mathscr{C}$ be just infinite. Then $\operatorname{Jac}\left(\mathfrak{A}_{G}\right)$ is either the zero ideal or equal to $\operatorname{Aug}\left(\mathfrak{A}_{G}\right)$.

Proof. Suppose that $\operatorname{Jac}\left(\mathfrak{A}_{G}\right) \neq\{0\}$. As the algebra $\mathfrak{A}_{G}$ is just infinite, it follows that $\operatorname{dim}_{F}\left(\mathfrak{A}_{G} / \operatorname{Jac}\left(\mathfrak{A}_{G}\right)\right)<\infty$. Since $|F|<\infty$, this implies $\left|\mathfrak{A}_{G} / \operatorname{Jac}\left(\mathfrak{A}_{G}\right)\right|<\infty$.

Now $\chi: F[G] \rightarrow \mathfrak{A}_{G}$ can be factored as

$$
F[G] \rightarrow F[\widetilde{G}] \rightarrow \mathfrak{A}_{G},
$$

where $\widetilde{G}$ is the closure of $G$ in the profinite $\operatorname{group} \operatorname{Aut}(T)$. Observe that $\widetilde{G}$ is a finitely generated pro- $p$ group. We obtain an induced group homomorphism

$$
\widetilde{\chi}: \widetilde{G} \rightarrow\left(\mathfrak{A}_{G} / \operatorname{Jac}\left(\mathfrak{A}_{G}\right)\right)^{*}
$$

from $\widetilde{G}$ to the unit group of the finite algebra $\mathfrak{A}_{G} / \operatorname{Jac}\left(\mathfrak{A}_{G}\right)$. Set $\widetilde{N}=\operatorname{ker} \widetilde{\chi}$, $N=G \cap \widetilde{N}$ and $I=\chi^{-1}\left(\operatorname{Jac}\left(\mathfrak{A}_{G}\right)\right)$. Then $\widetilde{N}$ is normal and of finite index in $\widetilde{G}$ and thus $\widetilde{N}$ is open in $\widetilde{G}$; see [7, Theorem 1.17]. Hence $\widetilde{G} / \widetilde{N}$ is a finite $p$-group. Consequently $G / N$ is a finite $p$-group.

As $\langle x-1 \mid x \in N\rangle \subseteq I$, the homomorphism $F[G] \rightarrow F[G] / I \cong \mathfrak{A}_{G} / \operatorname{Jac}\left(\mathfrak{A}_{G}\right)$ factors through $F[G / N]$. Since $G / N$ is a finite $p$-group, it follows that $\operatorname{Jac}(F[G / N])=\operatorname{Aug}(F[G / N])$ and therefore $\operatorname{Jac}\left(\mathfrak{A}_{G}\right)=\operatorname{Aug}\left(\mathfrak{A}_{G}\right)$.

Proposition 6.3 ([2, Proposition 4.22]). Let $G \in \mathscr{C}$ be just infinite. If $\mathfrak{A}_{G}$ is semiprimitive, then it is primitive.

Proof. Let $\mathcal{M}$ denote the collection of all maximal right ideals of $\mathfrak{A}_{G}$. Suppose that $\mathfrak{A}_{G}$ is semiprimitive, i.e., that $\bigcap_{M \in \mathcal{M}} M=\operatorname{Jac}\left(\mathfrak{A}_{G}\right)=0$. We need to produce an $M \in \mathcal{M}$ with $\operatorname{core}(M)=0$. For this it suffices to show that, if $M \in \mathcal{M}$ with $\operatorname{core}(M) \neq 0$, then $\operatorname{core}(M)=\operatorname{Aug}\left(\mathfrak{A}_{G}\right)$.

Let $M \in \mathcal{M}$ with $I=\operatorname{core}(M) \neq 0$. Since $\mathfrak{A}_{G}$ is just infinite and $|F|<\infty$, it follows that $\mathfrak{A}_{G} / I$ is finite. As in the previous proof, there is a normal subgroup $N$ of $G$ such that the epimorphism $F[G] \rightarrow \mathfrak{A}_{G} / I$ factors through the group algebra $F[G / N]$ of the finite $p$-group $G / N$.

Write $\bar{J}$ for the image of $J=\chi^{-1}(I) \subseteq F[G]$ in $F[G / N]$ and observe that $\bar{J}$ is a maximal two-sided ideal of $F[G / N]$. However in the finite local ring $F[G / N]$ the augmentation ideal $\operatorname{Aug}(F[G / N])$ is the only maximal two-sided ideal. Hence we obtain $\bar{J}=\operatorname{Aug}(F[G / N])$ and therefore $J=\operatorname{Aug}(F[G])$. Hence $I=\chi(J)=\operatorname{Aug}\left(\mathfrak{A}_{G}\right)$.

6.2. The depth function. Let $G=\left\langle a, \mathbf{b}^{(1)}, \ldots, \mathbf{b}^{(p)}\right\rangle \in \mathscr{C}$ be in standard form, acting on the regular $p$-adic rooted tree $T$ with vertices labelled by elements of $\bar{X}$. Recall that $\bar{X}$ consists of all words in the alphabet $X=$ $\{1, \ldots, p\}$, and the length of a word $\omega \in \bar{X}$ is denoted by $|\omega|$. Recall that every $g \in G$ can be expressed as $\left(g_{1}, \ldots, g_{p}\right) a^{\varepsilon_{a}(g)}$, where $\varepsilon_{a}(g) \in \mathbb{Z} / p \mathbb{Z}$ and 
$\left(g_{1}, \ldots, g_{p}\right) \in G^{p}$ is short for $\psi_{1}^{-1}\left(g_{1}, \ldots, g_{p}\right)$. Of course, the decomposition can be reiterated, giving $g_{\omega}=\left(g_{\omega 1}, \ldots, g_{\omega p}\right) a^{\varepsilon_{a}\left(g_{\omega}\right)}$ for any word $\omega \in \bar{X}$.

As in [25], we define a depth function

$$
\begin{aligned}
d: G \rightarrow \mathbb{N}_{0}, \quad d(g)=\min \left\{d \in \mathbb{N}_{0} \mid\right. & \forall \omega \in \bar{X} \text { with }|\omega| \geq d: \\
& \left.\left(g_{\omega 1}, \ldots, g_{\omega p}\right) \in\left\langle\mathbf{b}^{(1)}\right\rangle \cup \ldots \cup\left\langle\mathbf{b}^{(p)}\right\rangle\right\} .
\end{aligned}
$$

We remark that the function is well-defined, because by Lemma 3.10 for any given $g \in G$ the lengths $\partial\left(g_{\omega}\right)$ decrease down to 0 or 1 as $|\omega| \rightarrow \infty$. Furthermore, we observe for every $g \in G$ :

$\circ d(g)=d\left(\left(g_{1}, \ldots, g_{p}\right)\right)$,

$\circ d(g)=0$ if and only if $g \in\left\langle\mathbf{b}^{(1)}\right\rangle\langle a\rangle \cup \ldots \cup\left\langle\mathbf{b}^{(p)}\right\rangle\langle a\rangle$,

$\circ$ if $d(g) \neq 0$, then $d(g)=\max \left\{d\left(g_{i}\right) \mid 1 \leq i \leq p\right\}+1$.

We notice that the natural embedding of groups

$$
G \hookrightarrow(G \times \stackrel{p}{.} \times G) \rtimes\langle a\rangle, \quad g \mapsto\left(\left(g_{1}, \ldots, g_{p}\right), a^{\varepsilon_{a}(g)}\right)
$$

induces a natural embedding of $F$-algebras

$$
\mathfrak{A}_{G} \hookrightarrow\left(\mathfrak{A}_{G} \times \stackrel{p}{.} \times \mathfrak{A}_{G}\right) \rtimes F\langle a\rangle,
$$

where each $v$ of $\mathfrak{A}_{G}$ is mapped to $v_{0}+v_{1} a+\ldots+v_{p-1} a^{p-1}$ with $v_{i}=$ $\left(v_{i, 1}, \ldots, v_{i, p}\right) \in \mathfrak{A}_{G} \times \stackrel{p}{.} \times \mathfrak{A}_{G}$ for $0 \leq i \leq p-1$.

Let $\left(\mathfrak{A}_{G} \times \stackrel{p}{.} \times \mathfrak{A}_{G}\right)^{\circ}$ denote the image of $F\left\langle\mathbf{b}^{(1)}\right\rangle \cup \ldots \cup F\left\langle\mathbf{b}^{(p)}\right\rangle \subseteq F[G]$ in $\mathfrak{A}_{G} \times \stackrel{p}{.} \times \mathfrak{A}_{G}$. Though as the map $\chi$ from Section 6.1 is injective on $G$, we will often identify elements of $G$ with their images in $\mathfrak{A}_{G}$.

The depth function $d: G \rightarrow \mathbb{N}_{0}$ now extends to $\mathfrak{A}_{G}$ as follows: for $v=$ $v_{0}+v_{1} a+\ldots+v_{p-1} a^{p-1} \in \mathfrak{A}_{G}$, where $v_{i}=\left(v_{i, 1}, \ldots, v_{i, p}\right) \in \mathfrak{A}_{G} \times \stackrel{p}{.} . \times \mathfrak{A}_{G}$ for $1 \leq i \leq p-1$, we define recursively

$$
\begin{aligned}
d(v)=\max \left(\{ 0 \} \cup \left\{d\left(v_{i, j}\right)+1 \mid 0 \leq\right.\right. & i \leq p-1 \text { and } 1 \leq j \leq p \\
& \text { such that } \left.\left.v_{i} \notin\left(\mathfrak{A}_{G} \times \stackrel{p}{ } \times \mathfrak{A}_{G}\right)^{\circ}\right\}\right) .
\end{aligned}
$$

We observe that if $v=v_{0}$ has $d(v) \neq 0$, then $d(v)>d\left(v_{0 j}\right)$ for $1 \leq j \leq p$.

6.3. Invertibility. With reference to Remark 1.3 , let $H=\langle a, b\rangle$ be a generalised Gupta-Sidki group acting on the regular $p$-adic rooted $T$; recall that $a$ denotes the rooted automorphism of order $p$ and $b$ a directed automorphism defined recursively by

$$
\psi_{1}(b)=\left(a^{e_{1}}, \ldots, a^{e_{p-1}}, b\right), \text { where }\left\{e_{1}, \ldots, e_{p-1}\right\}=\{1, \ldots, p-1\} .
$$

Let $G=\left\langle a, \mathbf{b}^{(1)}, \ldots, \mathbf{b}^{(p)}\right\rangle \in \mathscr{C}$ be in standard form and just infinite. Suppose that $H \leq G$, and say, without loss of generality, that $b=b_{1}^{(1)}$. We denote by $\mathfrak{T}$ the tree enveloping algebra of the Sylow-pro- $p$ subgroup $S \leq \operatorname{Aut}(T)$ described in (2.1) and write $\mathfrak{A}_{G} \subseteq \mathfrak{T}$ for the tree enveloping algebra of $G$, as usual. 
For $v \in \mathfrak{T}$, we set $v^{[1]}=(v, . \underline{p}, v) \in \mathfrak{T} \times \stackrel{p}{.} \times \mathfrak{T} \subseteq \mathfrak{T}$ and recursively, for $i \geq 2$,

$$
v^{[i]}=\left(v^{[i-1]}, . . . ., v^{[i-1]}\right) \in \mathfrak{T} \times \stackrel{p}{i} .^{i} \times \mathfrak{T} \subseteq \mathfrak{T} .
$$

Further, identifying elements of $G$ with their images in $\mathfrak{A}_{G}$, we introduce the notation

$$
\begin{aligned}
& a_{*}=(a-1)^{p-1}=1+a+\ldots+a^{p-1} \in \mathfrak{A}_{G}, \\
& b_{*}=(b-1)^{p-1}=1+b+\ldots+b^{p-1} \in \mathfrak{A}_{G} .
\end{aligned}
$$

Observe that for $\left(v_{1}, \ldots, v_{p}\right) \in \mathfrak{T} \times . \underline{p} \times \mathfrak{T} \subseteq \mathfrak{T}$,

$$
a_{*}\left(v_{1}, \ldots, v_{p}\right) a_{*}=\left(v_{1}+\ldots+v_{p}\right)^{[1]} a_{*} .
$$

In the following proof, the idea of the first half comes from Sidki 25, Proposition 5.2], and was already used by Vieira [27, Theorem 1] for the special case of the generalised Gupta-Sidki group.

Lemma 6.4. Let $G=\left\langle a, \mathbf{b}^{(1)}, \ldots, \mathbf{b}^{(p)}\right\rangle \in \mathscr{C}$ be just infinite, and suppose that $\langle a, b\rangle$, for $b=b_{1}^{(1)}$ as in 6.1), is a generalised Gupta-Sidki group. Then $1+b a_{*}$ is not invertible in the tree enveloping algebra $\mathfrak{A}_{G}$.

Proof. Let $\eta=1+b a_{*}$. We suppose that $\eta$ is invertible in the tree enveloping algebra $\mathfrak{T}$ of the Sylow-pro-p subgroup $S \leq \operatorname{Aut}(T)$. Then, as the $a \mapsto a$ and $b \mapsto b^{-1}$ induce an automorphism of $\bar{G}$, we see that $\mu=1+b^{-1} a_{*}$ is also invertible in $\mathfrak{T}$. By [27, Lemma 2], we may express the inverses as

$$
\eta^{-1}=1-\rho a_{*} \quad \text { and } \quad \mu^{-1}=1-\sigma a_{*},
$$

where $\rho, \sigma \in \mathfrak{T} \times \stackrel{p}{.} \times \mathfrak{T} \subseteq \mathfrak{T}$ such that

$$
\rho\left(b+a_{*}\right)^{[1]}=b \quad \text { and } \quad \sigma\left(b^{-1}+a_{*}\right)^{[1]}=b^{-1} .
$$

Multiplying on the right by $\left(\mu^{-1}\right)^{[1]}=\left(1-\sigma a_{*}\right)^{[1]}$, we obtain from the first equation in (6.3),

$$
\rho b^{[1]}=\rho(b \mu)^{[1]}\left(\mu^{-1}\right)^{[1]}=b\left(1-\sigma a_{*}\right)^{[1]}
$$

and hence

$$
\rho=b\left(1-\sigma a_{*}\right)^{[1]}\left(b^{-1}\right)^{[1]} .
$$

Similarly, we deduce

$$
\sigma=b^{-1}\left(1-\rho a_{*}\right)^{[1]} b^{[1]} .
$$

Substituting (6.5) in (6.4) gives

$$
\rho=b\left(b^{-1}\right)^{[1]}-b\left(b^{-1}\right)^{[1]}\left(1-\rho a_{*}\right)^{[2]} b^{[2]} a_{*}^{[1]}\left(b^{-1}\right)^{[1]} .
$$

Assume for a contradiction that $\eta^{-1} \in \mathfrak{A}_{G}$, and hence $\rho=\left(\rho_{1}, \ldots, \rho_{p}\right) \in$ $\mathfrak{A}_{G} \times . \stackrel{p}{.} \times \mathfrak{A}_{G}$. Recalling the depth function $d$, we observe that $d(\rho) \geq d\left(\rho_{p}\right)$, where $\rho_{p}$ can be expressed according to (6.6) as

$$
\begin{aligned}
\rho_{p} & =1-\left(1-\rho a_{*}\right)^{[1]} b^{[1]} a_{*} b^{-1} \\
& =1-\left(1-\rho a_{*}\right)^{[1]} b^{[1]}\left(b^{-1}+b^{-a^{-1}} a+\ldots+b^{-a} a^{p-1}\right) .
\end{aligned}
$$


Now writing $\rho_{p}=v_{0}+v_{1} a+\ldots+v_{p-1} a^{p-1}$ with $v_{i} \in \mathfrak{A}_{G} \times \stackrel{p}{.} . \times \mathfrak{A}_{G}$ for $0 \leq i \leq p-1$, we obtain $d\left(\rho_{p}\right) \geq d\left(v_{0}\right)$, where

$$
v_{0}=1-\left(1-\rho a_{*}\right)^{[1]} b^{[1]} b^{-1}
$$

Writing $v_{0}=\left(v_{0,1}, \ldots, v_{0, p}\right)$, we see that $v_{0, p}=\rho a_{*}$ and hence $d\left(v_{0}\right) \geq$ $d\left(v_{0, p}\right)=d(\rho)$. The inequalities $d(\rho) \geq d\left(\rho_{p}\right) \geq d\left(v_{0}\right) \geq d(\rho)$ imply equality throughout and hence $d(\rho)=0$ which is equivalent to $\rho \in F\left\langle\mathbf{b}^{(j)}\right\rangle \subseteq \mathfrak{A}_{G}$ for some $j \in\{1, \ldots, p\}$. Recall that as before we identify $F\left\langle\mathbf{b}^{(j)}\right\rangle$ with its image in $\mathfrak{A}_{G}$.

If $j=1$, then $\rho=\rho_{p}=v_{0}=v_{0, p}=\rho a_{*}$, and therefore $\rho=0$, giving us the required contradiction. Now suppose that $j \neq 1$. According to (6.6), the coordinates of $\rho=\left(\rho_{1}, \ldots, \rho_{p}\right)$ are

$$
\begin{aligned}
& \rho_{i}=a^{e_{i}} b^{-1}-a^{e_{i}} b^{-1}\left(1-\rho a_{*}\right)^{[1]} b^{[1]} a_{*} b^{-1} \quad \text { for } 1 \leq i \leq p-1, \\
& \rho_{p}=1-\left(1-\rho a_{*}\right)^{[1]} b^{[1]} a_{*} b^{-1} .
\end{aligned}
$$

Moreover, $\rho \in F\left\langle\mathbf{b}^{(j)}\right\rangle$ implies that $\rho_{p-j+1} \in F\left\langle\mathbf{b}^{(j)}\right\rangle$ and $\rho_{1}, \ldots, \rho_{p-j}, \rho_{p-j+2}$, $\ldots, \rho_{p} \in F\langle a\rangle$. As $\rho_{1}, \ldots, \rho_{p-1}$ all differ by a left multiple of $a$, it follows that $\rho_{p-j+1} \in F\left\langle\mathbf{b}^{(j)}\right\rangle \cap F\langle a\rangle=F$. Consequently, there is a $\lambda \in F$ such that

$$
a^{e_{p-j+1}} b^{-1}\left(1-\left(\eta^{-1} b\right)^{[1]} a_{*} b^{-1}\right)=\lambda
$$

Our next aim is to express (6.7) in matrix form via the embedding

$$
\varphi: \mathfrak{A}_{G} \rightarrow \operatorname{Mat}_{p}\left(\mathfrak{A}_{G}\right)
$$

induced by

$$
a \mapsto\left(\begin{array}{ccccc}
0 & 1 & 0 & \ldots & 0 \\
0 & 0 & 1 & \ldots & 0 \\
\vdots & & \ddots & \ddots & \vdots \\
0 & \ldots & \ldots & 0 & 1 \\
1 & 0 & \ldots & \ldots & 0
\end{array}\right) \quad \text { and } \quad\left(c_{1}, \ldots, c_{p}\right) \mapsto\left(\begin{array}{ccccc}
c_{1} & & & & \\
& c_{2} & & & \\
& & \ddots & & \\
& & & c_{p-1} & \\
& & & c_{p}
\end{array}\right)
$$

cf. [2, Section 3]. First we compute the relevant terms individually: 


$$
\begin{aligned}
& \varphi\left(a^{p-j+1}\right)=\left(\begin{array}{cc}
0 & \operatorname{Id}_{j-1} \\
\operatorname{Id}_{p-j+1} & 0
\end{array}\right), \quad \varphi\left(a_{*}\right)=\left(\begin{array}{ccc}
1 & \cdots & 1 \\
\vdots & \ddots & \vdots \\
1 & \ldots & 1
\end{array}\right) \\
& \varphi\left(b^{-1}\right)=\left(\begin{array}{ccccc}
a^{-e_{1}} & & & & \\
& a^{-e_{2}} & & & \\
& & \ddots & & \\
& & & a^{-e_{p-1}} & \\
& & & & b^{-1}
\end{array}\right) \\
& \varphi\left(\left(\eta^{-1} b\right)^{[1]}\right)=\left(\begin{array}{ccccc}
\eta^{-1} b & & & & \\
& \eta^{-1} b & & & \\
& & \ddots & & \\
& & & \eta^{-1} b & \\
& & & & \eta^{-1} b
\end{array}\right) \text {. }
\end{aligned}
$$

Combining these terms, we deduce from 6.7),

$$
\begin{aligned}
& \lambda \operatorname{Id}_{p}=\varphi\left(a^{e_{p-j+1}} b^{-1}\left(1-\left(\eta^{-1} b\right)^{[1]} a_{*} b^{-1}\right)\right)= \\
& \quad\left(\begin{array}{cccc}
-a^{-e_{p-j+2}} \eta^{-1} b a^{-e_{1}} & \ldots & -a^{-e_{p-j+2}} \eta^{-1} b a^{-e_{p-1}} & -a^{-e_{p-j+2}} \eta^{-1} \\
\vdots & & \vdots & \vdots \\
-b^{-1} \eta^{-1} b a^{-e_{1}} & \ldots & -b^{-1} \eta^{-1} b a^{-e_{p-1}} & b^{-1}\left(1-\eta^{-1}\right) \\
a^{-e_{1}}\left(1-\eta^{-1} b a^{-e_{1}}\right) & \ldots & -a^{-e_{1}} \eta^{-1} b a^{-e_{p-1}} & -a^{-e_{1}} \eta^{-1} \\
\vdots & & \vdots & \vdots \\
-a^{-e_{p-j+1}} \eta^{-1} b a^{-e_{1}} & \ldots & -a^{-e_{p-j+1}} \eta^{-1} b a^{-e_{p-1}} & -a^{-e_{p-j+1}} \eta^{-1}
\end{array}\right) .
\end{aligned}
$$

Comparing the first and last terms of the first row, we see that $\lambda=0$. Hence $\rho=0$, and therefore $\eta^{-1}=1$, which cannot be possible. Thus, we have our contradiction.

Remark 6.5. In [18, Theorem 12.3] Passman claims that Sidki's proof [25] for the Gupta-Sidki 3-group follows through for Gupta-Sidki p-groups for all primes $p \geq 3$. However, (6.3) does not hold for Gupta-Sidki $p$-groups, where $p \geq 5$. One instead obtains the following equation

$$
\rho\left(b+a+a^{-1}+p-1\right)^{[1]}=b
$$

which prevents us from going on to the next deduction, as $a+a^{-1}+p-1 \neq a_{*}$. We do not know of a way to skirt around this.

Proposition 6.6. Let $G=\left\langle a, \mathbf{b}^{(1)}, \ldots, \mathbf{b}^{(p)}\right\rangle \in \mathscr{C}$ be just infinite, and suppose that $\langle a, b\rangle$, for $b=b_{1}^{(1)}$ as in (6.1), is a generalised Gupta-Sidki group. Then $\operatorname{Jac}\left(\mathfrak{A}_{G}\right)=0$. 
Proof. As $\operatorname{Jac}\left(\mathfrak{A}_{G}\right)$ is a quasi-invertible ideal and as $a_{*} \in \operatorname{Aug}\left(\mathfrak{A}_{G}\right)$, it suffices by Corollary 6.2, to prove that $1+\lambda a_{*}$ is not invertible in $\mathfrak{A}_{G}$ for some $\lambda \in \mathfrak{A}_{G}$. The statement of the proposition now follows from Lemma 6.4.

6.4. Graded ideals. Let $G=\left\langle a, \mathbf{b}^{(1)}, \ldots, \mathbf{b}^{(p)}\right\rangle \in \mathscr{C}$ be in standard form and just infinite. By conjugation, we may assume that $r_{1} \neq 0$ and we write $b=b_{1}^{(1)}$. We observe that the elements $a_{*}, b_{*} \in \mathfrak{A}_{G}$ defined in 6.2 satisfy $a_{*}^{2}=b_{*}^{2}=0$. Furthermore we write $b_{*}=\left(a_{*} \epsilon_{1}, \ldots, a_{*} \epsilon_{p-1}, b_{*}\right)$ where for $1 \leq i \leq p-1$,

$$
\epsilon_{i}= \begin{cases}0 & \text { if } e_{i}=0 \text { in } \mathbb{Z} / p \mathbb{Z}, \\ 1 & \text { otherwise }\end{cases}
$$

Several preliminary results will be needed; the first is by Smoktunowicz.

Theorem 6.7 ([2, Lemma 4.24] and [26, Theorem 1.1]). Let $\mathfrak{I}=\oplus_{i=1}^{\infty} \mathfrak{I}_{i}$ be a graded algebra (without unit) generated in degree 1 . Then the following are equivalent:

(1) $\operatorname{Jac}(\mathfrak{I})=\mathfrak{I}$;

(2) for every choice of $n, m \in \mathbb{N}$, all $n \times n$ matrices with entries in $\mathfrak{I}_{m}$ are nilpotent.

In the following, the proof is a slight modification of that in 25, Proposition 4.1.1], due to the allowance of arbitrary exponents $e_{i}$.

Proposition 6.8. Let $G=\left\langle a, \mathbf{b}^{(1)}, \ldots, \mathbf{b}^{(p)}\right\rangle \in \mathscr{C}$ be just infinite. The subalgebra $F\left[b_{*} a_{*}\right]$ of $\mathfrak{A}_{G}$ generated by $b_{*} a_{*}$ is a polynomial algebra. In particular, $b_{*} a_{*}$ is not nilpotent, and $\operatorname{Aug}\left(\mathfrak{A}_{G}\right)$ is not nil.

Proof. We denote $b_{*} a_{*}$ by $X$ and $a_{*} b_{*}$ by $Y$. Let

$$
N=\mid\left\{e_{i} \mid e_{i} \neq 0 \text { for } 1 \leq i \leq p-1\right\} \mid,
$$

regarded as an element of $(\mathbb{Z} / p \mathbb{Z})^{*}$. By induction,

$$
\begin{aligned}
X^{2 j-1} & =N^{j-1}\left(\epsilon_{1} Y^{j-1} a_{*}, \ldots, \epsilon_{p-1} Y^{j-1} a_{*}, X^{j-1} b_{*}\right) a_{*} \\
X^{2 j} & =N^{j-1}\left(\epsilon_{1} Y^{j}, \ldots, \epsilon_{p-1} Y^{j}, N X^{j}\right) a_{*}
\end{aligned}
$$

for all $j \geq 1$.

For a contradiction, assume that $X$ is a root of some non-zero polynomial $f \in F[t]$ of minimal degree $m=\operatorname{deg}(f) \in \mathbb{N}$, say. Note that $f$ has zero constant term, as otherwise it would imply that $X=b_{*} a_{*}$ were invertible. Now

$$
0=f(X)=\left(l_{1}, \ldots, l_{p-1}, q\right) a_{*}
$$

for some $l_{1}, \ldots, l_{p-1}, q \in \mathfrak{A}_{G}$, and from 6.9 we deduce that

$$
0=q=q_{0}(X)+q_{1}(X) b_{*},
$$

where $q_{0}, q_{1} \in F[t]$ satisfy $\max \left\{\operatorname{deg}\left(q_{0}\right), \operatorname{deg}\left(q_{1}\right)\right\}=\lfloor m / 2\rfloor$, and the polynomial $q_{0}$ also has zero constant term.

Now since $0=q X=q_{0}(X) X$ and $0=q a_{*}=q_{1}(X) X$, it follows that $X$ is a root of each of the polynomials $q_{0}(t) t, q_{1}(t) t$. We conclude that $m \in\{1,2\}$ 
by minimality. Thus $f$ is either $t$ or $t^{2}+c t$ for some constant $c \in F$. It follows by a direct computation, with the aid of $(6.9)$, that all these cases lead to a contradiction.

Proposition 6.9. Let $G=\left\langle a, \mathbf{b}^{(1)}, \ldots, \mathbf{b}^{(p)}\right\rangle \in \mathscr{C}$ be in standard form and just infinite. If $\operatorname{Aug}\left(\mathfrak{A}_{G}\right)$ is a graded algebra with the elements $a-1$ and $b_{i}^{(j)}-1$ for $1 \leq j \leq p, 1 \leq i \leq r_{j}$ being homogeneous, then $\operatorname{Jac}\left(\mathfrak{A}_{G}\right)=0$.

Proof. It follows that $X=b_{*} a_{*}$ is homogeneous of degree $m$, say. Proposition 6.8 shows that condition (2) of Theorem 6.7 does not hold for $n=1$. Therefore we obtain $\operatorname{Jac}\left(\operatorname{Aug}\left(\mathfrak{A}_{G}\right)\right)<\operatorname{Aug}\left(\mathfrak{A}_{G}\right)$.

Now it follows from Corollary 6.2 that $\operatorname{Jac}\left(\mathfrak{A}_{G}\right)=\operatorname{Aug}\left(\mathfrak{A}_{G}\right)$ or $\operatorname{Jac}\left(\mathfrak{A}_{G}\right)=$ 0 . However as $\operatorname{Jac}\left(\operatorname{Jac}\left(\mathfrak{A}_{G}\right)\right)=\operatorname{Jac}\left(\mathfrak{A}_{G}\right)$ (see [15, Exercise 4.7]), we must have $\operatorname{Jac}\left(\mathfrak{A}_{G}\right)=0$.

We can now give a proof of our main result.

Proof of Theorem 1.5. From Proposition 6.6 and Proposition 6.9 it follows that $\operatorname{Jac}\left(\mathfrak{A}_{G}\right)=0$ in both cases. From the proof of Corollary 6.2, we see that this ensures the existence of a maximal right ideal $M$, with null core, which gives us a faithful irreducible representation of $\mathfrak{A}_{G}$ on the $F$-space $\mathfrak{A}_{G} / M$.

6.5. Irreducible representations. We prove that when a branch generalised multi-edge spinal group has a non-trivial irreducible representation, then it has infinitely many. The proof is similar to but somewhat conciser than that of [19, Theorem 2.2].

Theorem 6.10. Let $G \in \mathscr{C}_{\text {reg }}$ and let $F$ be an algebraically closed field of characteristic $p$. If the group algebra $F[G]$ has at least one non-trivial irreducible module $M$, then $F[G]$ has infinitely many such irreducible modules.

Proof. For notational convenience, set $H=\gamma_{3}(G)$. Furthermore set $L=$ $\gamma_{3}\left(\operatorname{Stab}_{G}(1)\right)$, the normal finite-index subgroup of $H$ with $L \cong H \times \stackrel{p}{.} \times H$, by Proposition 3.3 . If $V_{1}, \ldots, V_{p}$ are irreducible $F[H]$-modules, then $V_{1} \otimes$ $\ldots \otimes V_{p}$ is an irreducible $F[L]$-module; see [23, Proposition 8.4.2]. Let $F_{0}$ denote the trivial 1-dimensional module, for both $F[G]$ and $F[H]$.

We consider $F[H]$. Let $U$ be the given non-trivial irreducible $F[G]$ module. Its restriction $U_{H}=U_{1}^{\prime} \oplus \ldots \oplus U_{t}^{\prime}$ is a finite direct sum of irreducible $F[H]$-modules, by Clifford theory. If all $U_{i}^{\prime} \cong F_{0}$, then $U$ is an irreducible $F[G / H]$-module, as $H$ acts trivially on $U$. But $G / H$ is a finite $p$-group and as the characteristic of $F$ is $p$, this implies that $U=F_{0}$, a contradiction. Therefore $F[H]$ has a non-trivial irreducible module $V$, and by [19, Lemma 2.1] it suffices to show that $H$ has infinitely many such.

By the branching property of $H$, we have, for all $m \in \mathbb{N}$, a subgroup $L_{m} \leq \operatorname{Stab}_{H}(m)$ which is isomorphic to $H \times p^{m} . \times H$ under $\psi_{m}$. We write $L_{m}=L_{m, 1} \times \ldots \times L_{m, p^{m}}$, where $L_{m, i} \cong H$ for $1 \leq i \leq p^{m}$. The group $G$ 
permutes these factors by conjugation. By viewing $V$ as a module for each $F\left[L_{m, i}\right]$, we can consider the $p^{m}+1$ irreducible $F\left[L_{m}\right]$-modules

$$
Y_{j}=V \otimes \stackrel{j}{j} . \otimes V \otimes F_{0} \otimes p^{m} \cdots^{j} \otimes F_{0},
$$

where $j \in\left\{0, \ldots, p^{m}\right\}$. Since $V \nsubseteq F_{0}$ and $G$ permutes the factors $L_{m, i}$ of $L_{m}$, it follows that $Y_{0}, \ldots, Y_{p^{m}}$ are in distinct orbits under the action of $G$.

For each $j$, let $Z_{j}$ be an irreducible constituent of $Y_{j}^{H}$. Since the $Y_{j}$ are in distinct $H$-orbits, it follows that $Z_{0}, \ldots, Z_{p^{m}}$ are distinct irreducible $F[H]$ modules. As this is true for all $m \in \mathbb{N}$, the result follows with the aid of [19, Lemma 2.1].

Acknowledgement. The second author was funded by the Alexander von Humboldt Foundation. We thank Amaia Zugadi-Reizabal for helpful remarks, e.g., concerning the generalised Gupta-Sidki groups. We are grateful to Alejandra Garrido for discussions on the EGS groups and for pointing out a lack of detail in the proof of [1, Proposition 5.2]. Finally we thank the anonymous referee for their detailed comments which led to significant improvements in the exposition of our paper.

\section{REFERENCES}

[1] T. Alexoudas, B. Klopsch and A. Thillaisundaram, Maximal subgroups of multi-edge spinal groups, Groups Geom. Dyn. 10 (2016), 619-648.

[2] L. Bartholdi, Branch rings, thinned rings, tree enveloping rings, Israel J. Math. 154 (2006), 93-139.

[3] L. Bartholdi, Branch rings, thinned rings, tree enveloping rings - Erratum, Israel J. Math. 193 (1) (2013), 507-508.

[4] L. Bartholdi, R. I. Grigorchuk and Z. Šuniḱ, Branch groups, in Handbook of algebra Volume 3, pp. 98-1112 (North-Holland, Amsterdam, 2003).

[5] I. V. Bondarenko, Finite generation of iterated wreath products, Arch. Math. (Basel) 95 (2010), 301-308.

[6] K. Bou-Rabee, P.-H. Leemann and T. Nagnibeda, Weakly maximal subgroups in regular branch groups, J. Algebra 455 (2016), 347-357.

[7] J. D. Dixon, M. P. F. du Sautoy, A. Mann, and D. Segal, Analytic pro-p groups (Cambridge University Press, Cambridge, 1999).

[8] M. Ershov and A. Jaikin-Zapirain, Groups of positive weighted deficiency and their applications, J. Reine Angew. Math. 677 (2013), 71-134.

[9] G. A. Fernández-Alcober, A. Garrido and J. Uria-Albizuri, On the congruence subgroup property for GGS-groups, to appear in Proc. Amer. Math. Soc., preprint: arXiv:1604.03465.

[10] G. A. Fernández-Alcober and A. Zugadi-Reizabal, GGS-groups: order of congruence quotients and Hausdorff dimension, Trans. Amer. Math. Soc. 366 (2014), 1993-2017.

[11] D. Francoeur and A. Garrido, Maximal subgroups of groups of intermediate growth, preprint: arXiv:1611.01216.

[12] A. Garrido, On the congruence subgroup problem for branch groups, Israel J. Math. 216 (2016), 1-13.

[13] R. I. Grigorchuk, Just infinite branch groups, in New horizons in pro-p groups (ed. M. du Sautoy, D. Segal and A. Shalev), pp. 121-179 (Birkhäuser, Boston, 2000).

[14] R. I. Grigorchuk and J. S. Wilson, The uniqueness of the actions of certain branch groups on rooted trees, Geom. Dedicata 100 (2003), 103-116. 
[15] T. Y. Lam, A first course in noncommutative rings (Springer-Verlag, New York, 2001).

[16] V. D. Mazurov and E. I. Khukhro, The Kourovka Notebook, Unsolved problems in group theory, 18th edition, Russian Academy of Sciences, Siberian Division, Institute of Mathematics, Novosibirsk (2016), arXiv:1401.0300v9.

[17] D. S. Passman, The semiprimitivity of group algebras, in Methods in ring theory (Levico Terme, 1997), pp. 199-211 (Lecture Notes in Pure and Appl. Math. 198, Dekker, New York, 1998).

[18] D. S. Passman, Semiprimitivity of group algebras: Past results and recent progress, in Trends in Ring Theory: Proceedings of a Conference at Miskolc, Hungary (1996), CMS Conference Proceedings 22, pp. 127-157 (American Mathematical Society, Providence, RI, 1998).

[19] D. S. Passman and W. Temple, Representations of the Gupta-Sidki group, Proc. Amer. Math. Soc. 124 (1996), 1403-1410.

[20] E. L. Pervova, Maximal subgroups of some non locally finite p-groups, Internat. J. Algebra Comput. 15 (2005), 1129-1150.

[21] E. L. Pervova, Profinite completions of some groups acting on trees, J. Algebra 310 (2007), 858-879.

[22] E. L. Pervova, Profinite topologies in just infinite branch groups, preprint 2002-154 of the Max Planck Institute for Mathematics, Bonn, Germany.

[23] D. J. S. Robinson, A course in the theory of groups (Springer-Verlag, New YorkBerlin, 1982).

[24] A. V. Rozhkov, On the theory of groups of Aleshin type, Mat. Zametki 40 (1986), $572-589$.

[25] S. Sidki, A primitive ring associated to a Burnside 3-group, J. London Math. Soc. 55 (1997), 55-64.

[26] A. Smoktunowicz, The Jacobson radical of rings with nilpotent homogeneous elements, Bull. Lond. Math. Soc. 40 (6) (2008), 917-928.

[27] A. C. Vieira, Modular algebras of Burnside p-groups, Mathemática Contemporânea 21 (2001), 287-304.

[28] T. Vovkivsky, Infinite torsion groups arising as generalizations of the second Grigorchuk group, in Algebra (Moscow, 1998), pp. 357-377 (de Gruyter, Berlin, 2000).

Benjamin Klopsch: Mathematisches Institut, Heinrich-Heine-Universität, 40225 DÜsSELDORF, GERMANY

E-mail address: klopsch@math.uni-duesseldorf.de

Anitha Thillaisundaram: School of Mathematics and Physics, University OF LinCOLN, LinCOLN LN6 7TS, UK

E-mail address: anitha.t@cantab.net 